\title{
Depth-resolved investigation of the lateral magnetic correlations in a gradient nanocrystalline multilayer
}

\author{
Emmanuel Kentzinger, ${ }^{1}$ Ulrich Rücker, ${ }^{1}$ Boris Toperverg, ${ }^{1}$ Frédéric Ott, ${ }^{2}$ and Thomas Brückel ${ }^{1}$ \\ ${ }^{1}$ Institut für Festkörperforschung JCNS and JARA-FIT, Forschungszentrum Jülich GmbH, D-52425 Jülich, Germany \\ ${ }^{2}$ Laboratoire Léon Brillouin, CE-Saclay, F-91191 Gif-sur-Yvette, France
}

(Received 1 August 2007; published 26 March 2008)

\begin{abstract}
We present an investigation of the structural and magnetic correlations during the remagnetization process of a gradient nanocrystalline multilayer with uniform uniaxial magnetic anisotropy by specular reflectometry and off-specular scattering of polarized neutrons with polarization analysis. The scattering data were analyzed within the distorted wave Born approximation (DWBA) including interfacial roughness correlations and lateral correlations of the spin misalignment. The magnetization reversal proceeds sequentially from the bottommost and thinnest ferromagnetic layers to the topmost and thickest ones. It is quantitatively explained within the random anisotropy model including dominant uniform uniaxial anisotropy. Two types of lateral spin misalignment were deduced, random and not random: The first one in all layers due to the random orientation of the grains, the second one in the layers with nonreversed magnetizations only and due to the applied field acting against the uniaxial anisotropy.
\end{abstract}

DOI: 10.1103/PhysRevB.77.104435

PACS number(s): 75.25.+z, 61.05.fj, 75.50.Tt, 75.70.-i

\section{INTRODUCTION}

Over the past 20 years, the ability to build magnetic materials nanostructured along one dimension has led to a particularly interesting class of materials for both scientific and technological applications. Studies on subjects such as interlayer exchange coupling, giant magnetoresistance, colossal magnetoresistance, tunnel magnetoresistance, currentinduced magnetization switching, ${ }_{1}^{1}$ or exchange bias ${ }^{2}$ have led to the exciting possibility of utilizing electron spin for information processing.

The development of lithography $y^{3,4}$ and self-organization ${ }^{5,6}$ techniques has driven the research on samples nanostructured along several dimensions, such as "nanostripes" or "nanodots." Fundamentally, novel properties can be expected if the size of the structures becomes comparable to or smaller than certain characteristic length scales, such as the spin diffusion length, charge carrier mean free path, ferromagnetic exchange length, or Fermi wavelength., ${ }^{3,7}$ For example, magnetization reversal processes can be modified drastically in magnetic structures confined to sizes that preclude the domain wall formation. Also, the proximity of nanoelements can interfere with the spin diffusion length, a quantity that determines long range order phenomena in itinerant magnetism.

From the point of view of applications, magnetic nanostructures are the critical building blocks of important magnetoelectronics devices, such as the magnetic random access memory or patterned recording media. Due to the necessary miniaturization of such devices and the proximity of the superparamagnetic limit, ${ }^{8}$ the magnetic interaction between the neighboring cells ${ }^{9}$ is becoming a more and more important parameter that has to be understood and, hitherto, controlled. Also, it has recently been shown that the dilution of a small amount of magnetic transition metal atoms in a semiconductor matrix can lead to the self organization of well ordered, transition metal rich, magnetic nanocrystals. ${ }^{10,11} \mathrm{~A}$ strong magnetostatic or carrier-mediated coupling between the nanocrystals is expected, which could explain the high Curie temperature and strong magnetoresistance in certain diluted ferromagnetic semiconductors.

Specular reflection of polarized neutrons has been shown to be a method of choice for the quantitative, depth-resolved determination of the amplitudes and directions of the magnetizations in magnetic multilayers, leading to precious informations, for example on multilayers with interlayer exchange coupling, ${ }^{12,13}$ on exchange bias systems, ${ }^{14-16}$ on exchange spring magnets, ${ }^{17}$ on the magnetism of the interface between a ferromagnet and a superconductor, ${ }^{18,19}$ or on dipolarly coupled magnetic multilayers. ${ }^{20}$ In the specular reflection geometry the wave vector transfer $\mathbf{Q}$ of the radiation is perpendicular to the sample surface and only laterally averaged informations can be deduced.

In the off-specular geometry, $\mathbf{Q}$ has one or two of its components lying in the sample plane, which allows to probe lateral correlations of the magnetization fluctuations on length scales ranging from several tens of $\mu \mathrm{m}^{21-25}$ down to the $\mathrm{nm}$ range. ${ }^{26}$ Neutron scattering under grazing incidence with polarization analysis is a method of choice to determine lateral fluctuations in direction and modulus of the magnetization vector in devices relevant for magnetoelectronics, making it a unique probe, even compared to the powerful techniques of magnetic force microscopy, photoemission electron microscopy, and resonant $\mathrm{x}$-ray magnetic scattering or dichroism, as a depth information is available, which is not the case for the other methods mentioned.

Although the cross section of specular reflection of polarized neutrons has been modeled quite quickly after the first observations, ${ }^{27-29}$ the cross section for grazing incidence scattering of polarized neutrons with polarization analysis has received only recently a full derivation. ${ }^{30,31}$ At grazing incidence, the lengths obtained by projecting the coherence volume of the neutron beam on the sample surface may become rather large (up to the mm range) but usually remain smaller than the sample surface and therefore have to be compared with the lengths of lateral correlations present in 
the sample. This can have important consequences for the interpretation and simulation of specular reflectivity ${ }^{20}$ and off-specular scattering, ${ }^{13,32,33}$ as explained in Sec. II A. At grazing incidence, the measured off-specular scattering can also be affected strongly by dynamical effects such as refraction and total external reflection. Those phenomena, inherently dependent upon multiple scattering, are not reproduced by simulations within the Born approximation. In that case, the theoretical description has to be performed in the framework of the distorted wave Born approximation (DWBA).

To the authors knowledge, reflectivity and off-specular scattering of polarized neutrons with polarization analysis, coupled with state-of-the-art data analysis, is the only method that quantitatively and in a nonperturbing way allows depth-resolved investigations of the magnetizations and of the correlations between their in-plane fluctuations in buried layers.

In this paper, we show reflectivity and off-specular scattering of polarized neutrons and polarization analysis from an $\mathrm{FeCoV} / \mathrm{TiN}$ remanent neutron polarizing supermirror. ${ }^{34} \mathrm{~A}$ polarizing supermirror is a ferromagnetic/nonmagnetic multibilayer system with a gradient in the bilayer thicknesses. It is used for the polarization of cold and thermal neutron beams. Our interest in this system lies in the nanocrystallinity of the ferromagnetic layers with randomly oriented grains that are exchange coupled with their neighbors and that have sizes smaller than the ferromagnetic exchange length, leading to reduced coercivity of the ferromagnetic layers. ${ }^{35}$ Moreover, a well defined uniaxial magnetic anisotropy has been induced in this system, coherent over the whole surface of the sample. ${ }^{36}$ All this, together with the high saturation magnetization of the ferromagnetic layers makes $\mathrm{FeCoV}$ an excellent candidate for high-frequency thin film read-head material. ${ }^{37,38}$ In this supermirror, the lateral grain size increases with the layer thickness, ${ }^{39}$ leading to an increase of the coercive field from the thinnest to the thickest layers. ${ }^{34}$ The issue here is to figure out whether the evolution of the lateral grain size reflects itself in an evolution of the layer magnetization reversal mechanism and/or of the lateral magnetic correlations.

The paper is organized as follows. In Sec. II we describe the experimental geometry and sketch the results of the calculation of neutron specular reflectivity and scattering under grazing incidence with polarization analysis. We also describe the structure factors for the two types of fluctuations encountered here, namely bulk lateral magnetic fluctuations and interfacial structural roughnesses. The principle of a remanent polarizing supermirror is presented in Sec. III, the experimental apparatus and the data analysis procedure in Sec. IV. In Sec. V, the measurements on the supermirror are presented together with their analysis, including the specular reflectivity and the off-specular scattering. From the specular reflectivity, we deduce that the magnetization reversal proceeds sequentially from the bottommost and thinnest $\mathrm{FeCoV}$ layers to the topmost and thickest ones, we conclude on the presence of magnetically dead layers at both $\mathrm{FeCoV} / \mathrm{TiN}$ and $\mathrm{TiN} / \mathrm{FeCoV}$ interfaces, and deduce that the interfacial roughness increases from the bottom-most to the topmost interface in a nonlinear way. Those results are in agreement with other studies on the same system. The off-specular scat-

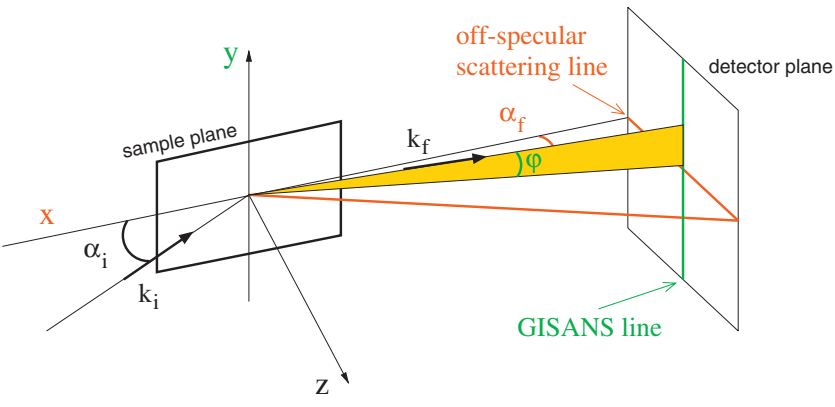

FIG. 1. (Color) Measurement geometry. On a reflectometer, like HADAS, the collimation of the beam is defined by two slits parallel to the $y$ axis. In that case, the measured intensities are not resolved but integrated along the $\varphi$ direction.

tering is strongly spin polarized and its origin can be separated into interfacial roughness with strong cross-correlations between interfaces and lateral correlations of the spin misalignment without any cross-correlations between the layers. Two types of lateral spin misalignments are deduced, random and not random, the first one being due to the random orientation of the grains, the second one due to the uniform uniaxial anisotropy. The results are discussed in Sec. VI. We quantitatively correlate the coercivity of the different $\mathrm{FeCoV}$ layers to their grain size changing with their thickness in the framework of the random anisotropy model (RAM) with dominant uniaxial anisotropy. We also discuss why we did not deduce any dependence of the lateral correlation length of the spin misalignment with grain size, in disagreement with the RAM. Finally, some concluding remarks and an outlook are given in Sec. VII.

\section{NEUTRON REFLECTIVITY AND SCATTERING UNDER GRAZING INCIDENCE WITH POLARIZATION ANALYSIS}

\section{A. Spatial coherence of the beam}

The measurement geometry is depicted in Fig. 1. Neutrons impinge on the sample surface with wave vector $\mathbf{k}_{i}$ under the grazing angle of incidence $\alpha_{i}$ and are detected with outgoing wave vector $\mathbf{k}_{f}$ whose direction is defined by the angles $\alpha_{f}$ and $\varphi$ in planes, respectively parallel and perpendicular to the plane of incidence defined by $\mathbf{k}_{i}$ and the normal to the sample surface. We will restrict our discussion to elastic scattering, i.e., $k_{i}=k_{f}=k=2 \pi / \lambda$, where $\lambda$ is the neutron wavelength.

In the present experimental geometry, the collimation of the beam is defined by two slits parallel to the $y$ axis. In that case, the detected signal is integrated over $\varphi$, scattering can be discriminated from the specular reflex along the offspecular line, and lateral correlations are probed along the $x$ direction. Therefore, if $\mathbf{Q}=\mathbf{k}_{f}-\mathbf{k}_{i}$ and $\mathbf{Q}_{\|}=\left(Q_{x}, Q_{y}\right)$ are the wave vector transfer and its projection on the sample plane respectively, then $Q_{x}$ is resolved and $Q_{y}$ is not.

An important quantity to consider in the interpretation and simulation of (specular) reflectivity and scattering under grazing incidence is the coherence volume of the neutron beam, because its projection $S_{\text {coh }}$ along the sample plane is 
usually much smaller than the (illuminated part of the) sample surface. $S_{\mathrm{coh}}=l_{x} \cdot l_{y}$ is related to the resolutions in the in-plane components of the momentum transfer $\left(l_{x}\right.$ $\left.\simeq 2 \pi / \delta Q_{x}, l_{y} \simeq 2 \pi / \delta Q_{y}\right)$. It depends on several factors such as the wavelength resolution, the beam collimation and the angles $\alpha_{i}, \alpha_{f}$, and $\varphi$. In the present experimental geometry $l_{x}$ ranges typically from several $\mu \mathrm{m}$ to several $\mathrm{mm}$ and, as $\delta Q_{y}$ is relaxed, $l_{y}$ is relatively small (several $\mathrm{nm}$ ). To be consistent, the projection $l_{\perp}$ of the coherence volume of the beam on the axis perpendicular to the sample surface has also to be considered. It is related to the component of $\mathbf{Q}$ perpendicular to the sample surface $\left(l_{\perp} \simeq 2 \pi / \delta Q_{\perp}\right)$ which can reach tens of $\mu \mathrm{m}$. Neutron waves scattered from objects separated in the z-direction by distances smaller than $l_{\perp}$ still interfere. In the case of supermirrors consisting of a stack of many layers resulting in a relatively large total thickness, special care has to be taken. In the case of the supermirror considered here, the total thickness is of the order of $1.5 \mu \mathrm{m}$. The reflectivity and diffuse scattering data were very well reproduced at all $\alpha_{i}$ and $\alpha_{f}$ angles by the simulations, assuming coherence over the whole penetration depth of the neutrons.

If, within the lateral coherence surface $S_{\text {coh }}$, the sample can be considered as homogeneous and flat, then no lateral momentum can be transferred, and intensity is seen only in the specular direction defined by $\mathbf{Q}_{\|}=0$ with an accuracy given by $\delta \mathbf{Q}_{\|}$. The measured reflectivity is an average over the different reflectivities from the different surfaces of coherence of the sample for which the homogeneous and flat approximation holds. If, on the contrary, the invariance of the system with respect to a lateral shift smaller than $l_{x}$ or $l_{y}$ is violated, lateral momentum transfer can occur and some intensity can be detected off-specular, i.e., at $\mathbf{Q}_{\|} \neq 0$. The measured scattering cross section is an average over the different scattering cross sections from the different coherence surfaces of the sample.

Usually, when spanning a whole range of $\alpha_{i}$ and $\alpha_{f}$ values, the off-specular signal $\left(\alpha_{i} \neq \alpha_{f}\right)$ coexists with specular reflectivity $\left(\alpha_{i}=\alpha_{f}\right)$. Specular reflectivity originates from the depth variation of the laterally averaged interaction potential between neutron and matter and off-specular scattering from the fluctuations around this mean value.

Note that a signal of scattering nature can be measured at the specular position. For a good interpretation of the measured data it is therefore important to measure both specular and off-specular signals. ${ }^{13,32,33}$

\section{B. Specular reflectivity}

In this section the calculation of the specular reflectivity with polarization analysis is presented within the recursion formalism of Parratt. ${ }^{40}$ We will also present how the inefficiencies of the polarizer and the analyzer as well as interfacial roughness are taken into account.

We assume that the sample is laterally homogeneous and flat, the neutron-matter interaction potential having a variation only along the depth of the sample. We subdivide the system in layers such that, in each layer, the neutron-matter interaction is a constant. The interaction potential operator in layer $l$ can be separated into two parts, i.e., $\hat{V}_{l}=V_{l}^{N} \hat{1}+\hat{V}_{l}^{M}$, where $V_{l}^{N}$ is the neutron-nucleus interaction potential and $\hat{V}_{l}^{M}=-\mu_{n} \hat{\boldsymbol{\sigma}} \mathbf{B}_{l}$ is the magnetic dipole interaction operator between the neutron magnetic moment operator $\mu_{n} \hat{\boldsymbol{\sigma}}$ and the magnetic induction $\mathbf{B}_{l}$. $\hat{\boldsymbol{\sigma}}$ is the vector of Pauli matrices, $\mu_{n}=\gamma \mu_{N}$ where $\mu_{N}$ is the nuclear magneton and $\gamma=-1.91$ is the neutron gyromagnetic factor. The interaction potential operator can be written as $\hat{V}_{l}=2 \pi \hbar^{2} / m\left(\rho_{l}^{N} \hat{1}+\rho_{l}^{M} \hat{\boldsymbol{\sigma}} \mathbf{b}_{l}\right)$ where $\rho_{l}^{N}$ and $\rho_{l}^{M}$ are the nuclear and magnetic scattering length densities, respectively, and $\mathbf{b}_{l}$ is the unit vector oriented along $\mathbf{B}_{l}$. $\mathbf{b}_{l}$ provides a quantization axis for the neutron spin. The two eigenvectors $|+\rangle$ and $|-\rangle$ of the operator $\hat{\boldsymbol{\sigma}} \mathbf{b}_{l}$ with eigenvalues +1 or -1 define states of the neutron with "+" or "-" spin projection along the quantization axis. The neutron wave function, solution of the Schrödinger equation in coordinates and spin space for the potential $\hat{V}_{l}$ and for an incoming plane wave with wave vector $\mathbf{k}_{i}$ and angle of incidence $\alpha_{i}$ is a linear combination of those two eigenstates. Inside each layer 1, it can be written in the following way:

$$
\left|\psi_{i l}\left(\mathbf{k}_{i}, \mathbf{r}\right)\right\rangle=e^{i \kappa_{i} \mathbf{r}} \cdot \hat{S}_{i l}(z) \cdot\left|\psi_{i 0}\left(\mathbf{k}_{i}, 0\right)\right\rangle,
$$

where $\boldsymbol{\kappa}_{i}$ is the in-plane (conserving) component of $\mathbf{k}_{i}$ and $\hat{S}_{i l}$ the propagation operator inside layer $l$ :

$$
\hat{S}_{i l}(z)=e^{i \hat{p}_{i l}\left(z-z_{l-1}\right)} \hat{t}_{i l}+e^{-i \hat{p}_{i l}\left(z-z_{l-1}\right)} \hat{r}_{i l},
$$

where $z_{l-1}$ is the height of the interface between layers $l-1$ and $l . z=0$ is the interface with vacuum $(l=0)$ and $\mathrm{z}$ is counted positive towards the substrate. $\hat{t}_{i l}$ and $\hat{r}_{i l}$ are the amplitude operators in spin space of the reflected and transmitted waves in layer $l$.

The component of the incident wave vector in layer $l$ perpendicular to the sample surface, operator in spin space, is given by $\hat{p}_{i l}=\sqrt{p_{i 0}^{2} \hat{1}-\hat{p}_{c, l}^{2}}$ where $p_{i 0}=k_{i} \sin \left(\alpha_{i}\right)$ is the component of $\mathbf{k}_{i}$ in vacuum perpendicular to the sample surface. The eigenvalues $\sqrt{4 \pi\left(\rho_{l}^{N}+\rho_{l}^{M}\right)}$ and $\sqrt{4 \pi\left(\rho_{l}^{N}-\rho_{l}^{M}\right)}$ of the operator $\hat{p}_{c, l}=\sqrt{4 \pi\left(\rho_{l}^{N} \hat{1}+\rho_{l}^{M} \hat{\boldsymbol{\sigma}} \mathbf{b}_{l}\right)}$ are the critical values of $p_{i 0}$ for total external reflection of spin "+" and spin "-" neutrons, respectively, at an interface between vacuum and a very thick layer of type 1, i.e., a layer of nuclear scattering length density $\rho_{l}^{N}$, magnetic scattering length density $\rho_{l}^{M}$, and magnetic field orientation $\mathbf{b}_{l} . \hat{p}_{i l}$ is diagonal when the quantization axis is taken along $\mathbf{b}_{l}$ with the eigenvalues $p_{i l}^{ \pm}$ $=\sqrt{p_{i 0}^{2}-4 \pi\left(\rho_{l}^{N} \mp \rho_{l}^{M}\right)}$. Like any function of a linear combination of the unit matrix and the Pauli matrices, $\hat{p}_{i l}$ can be written as another linear combination of the unit matrix and the Pauli matrices ${ }^{41,42}$ :

$$
\hat{p}_{i l}=\frac{1}{2}\left[\left(p_{i l}^{+}+p_{i l}^{-}\right) \hat{1}+\left(p_{i l}^{+}-p_{i l}^{-}\right) \hat{\boldsymbol{\sigma}} \mathbf{b}_{l}\right] .
$$

Solving the Schrödinger equation in each layer 1, and taking into account the continuity relations at the interfaces of the wave function and its first derivative perpendicular to the sample surface, the ratio $\hat{X}_{i l}=\hat{r}_{i l} \hat{t}_{i l}^{-1}$ of the amplitude operators of the reflected and transmitted waves in layer $l$ can be calculated based on the recursion formalism of Parratt. ${ }^{31,40,43,44}$ $\hat{X}_{i l}$ is given by 


$$
\begin{aligned}
\hat{X}_{i l}= & e^{i \hat{p}_{i l} d}\left[\left(\hat{1}-\hat{p}_{i l}^{-1} \hat{p}_{i l+1}\right)+\left(\hat{1}+\hat{p}_{i l}^{-1} \hat{p}_{i l+1}\right) \hat{X}_{i l+1}\right] \\
& \times\left[\left(\hat{1}+\hat{p}_{i l}^{-1} \hat{p}_{i l+1}\right)+\left(\hat{1}-\hat{p}_{i l}^{-1} \hat{p}_{i l+1}\right) \hat{X}_{i l+1}\right]^{-1} e^{i \hat{p}_{i l} d_{l}},
\end{aligned}
$$

where $d_{l}$ is the thickness of layer 1 .

The measured reflectivity $\mathcal{R}$ is deduced from the reflectance matrix $\hat{R}=\hat{r}_{i 0}$, i.e., the amplitude operator of the reflected wave in vacuum, if the amplitude operator of the impinging neutron wave is set to $\hat{t}_{i 0}=\hat{1}$ and a semi-infinite substrate is assumed $\left(\hat{r}_{i N+1}=\hat{0}\right)$. The reflectance matrix transforms the spin components of the incoming neutron wave into the spin components of the reflected wave. The measured reflectivity is the modulus squared of the matrix element of $\hat{R}$ between the bra and the ket representing the incoming and reflected waves, respectively, averaged over all the states prepared by the polarizer and accepted by the analyzer. Let $\mathbf{P}_{i}$ be the vector in coordinate space defining the spin-polarization (in magnitude and direction) of the incoming beam prepared by the polarizing device and $\mathbf{P}_{f}$ the corresponding vector characterizing the analyzer device. ${ }^{42}$ When the incoming beam is prepared in a pure state, i.e., with all neutrons having the same spin projection along the quantization axis defined by the guide field, then $\left|\mathbf{P}_{i}\right|=1$. When the pure state is the $|+\rangle(|-\rangle)$ state the component of $\mathbf{P}_{i}$ along that axis is equal to $+1(-1)$. In general, the polarizing device is not perfect and $\left|\mathbf{P}_{i}\right|<1$. The same argumentation holds for $\mathbf{P}_{f}$. The measured reflectivity then writes ${ }^{31,44}$

$$
\mathcal{R}=\left\langle\operatorname{Tr}\left(\hat{\rho}_{f} \hat{R} \hat{\rho}_{i} \hat{R}^{+}\right)\right\rangle,
$$

where the density matrices ${ }^{41,42} \hat{\rho}_{i}$ and $\hat{\rho}_{f}$ of the polarizer and the analyzer are deduced from the polarization vectors through the relation $\hat{\rho}_{i, f}=\frac{1}{2}\left(\hat{1}+\mathbf{P}_{i, f} \hat{\boldsymbol{\sigma}}\right)$. The bracket $\langle\cdot\rangle$ in the above equation stands for an average over all the coherence volumes probed by the beam (cf. Sec. II A).

Structural roughness between layer $l$ and $l+1$ is taken into account by assuming a Gaussian height distribution function of the interfaces of root-mean-squared width bounded to $\sigma_{l}$. Structural roughness alone does not produce any additional spin-flip process. Therefore, and following Nevot and Croce result $^{45-47}$ who found that the Fresnel reflection coefficient had to be corrected by a damping term, we use Eq. (5) again, substituting the operator $\left(\hat{1}-\hat{p}_{i l}^{-1} \hat{p}_{i l+1}\right)$ appearing two times in Eq. (4) by the operator $\left(\hat{1}-\hat{p}_{i l}^{-1} \hat{p}_{i l+1}\right) e^{\left(-2 \sigma_{l}^{2} \hat{p}_{i l} \hat{p}_{i l+1}^{R}\right)}$, where $\hat{p}_{i l}^{R}$ has the same functional form as $\hat{p}_{i l}$ but is now a function of $\hat{\boldsymbol{\sigma}} \mathbf{b}_{l}^{R}$, where $\mathbf{b}_{l}^{R}$ is the projection of $\mathbf{b}_{l}$ along the incoming polarization vector: $\mathbf{b}_{l}^{R}=\left(\mathbf{P}_{i} \cdot \mathbf{b}_{l}\right) \cdot \mathbf{P}_{i} /\left|\mathbf{P}_{i}\right|^{2}$.

\section{Scattering cross section within the DWBA}

If fluctuations of the height of interfaces, of the nuclear scattering length densities, or of the magnetic fields in the layers exist with correlations on length scales smaller than $l_{x}$, then off-specular scattering can be observed. In the grazing incidence geometry, the scattering can be strongly affected by dynamical effects such as refraction and total external reflection. For example, one can observe an enhancement of the off-specular scattering at $\alpha_{i}$ and $\alpha_{f}$ angles close to the

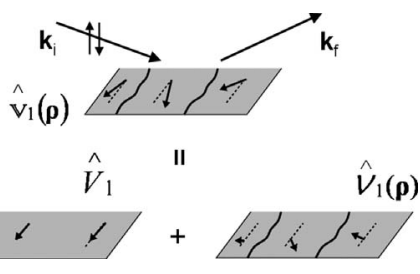

FIG. 2. Decomposition of the potential $\hat{V}_{l}(\boldsymbol{\rho})$ in layer $l$, within the DWBA. $\hat{V}_{l}(\boldsymbol{\rho})=\hat{V}_{l}+\hat{V}_{l}(\boldsymbol{\rho})$, where $\hat{V}_{l}$ is the reference potential leading to specular reflectivity and $\hat{\mathcal{V}}_{l}(\boldsymbol{\rho})$ is the residual one leading to off-specular scattering. $\hat{V}_{l}$ is constant inside layer $l$ and $\hat{\mathcal{V}}_{l}(\boldsymbol{\rho})$ is fluctuating laterally. $\mathbf{k}_{i}\left(\mathbf{k}_{f}\right)$ is the wave vector of the incident (outgoing) plane wave outside the sample.

critical angle of total reflection, the so-called Yoneda wings. ${ }^{48}$ In that range of angles, formulating the scattering cross section within the Born approximation fails in reproducing the measured data. The DWBA is used when the Hamiltonian can be decomposed into the sum of a reference Hamiltonian that can be solved exactly, plus a residual potential that can be regarded as a perturbation. In the form of the DWBA introduced by Vineyard ${ }^{49}$ and Sinha et al. ${ }^{47}$ and later on by Pynn ${ }^{50}$ and Holy et al..$^{51,52}$ for reflectivity and off-specular scattering from rough interfaces, the reference Hamiltonian corresponds to the system with flat interfaces leading to specular reflection (see Sec. II B). In the case of multilayers with micromagnetic fluctuations like, e.g., magnetic domains, the reference Hamiltonian inside each layer consists in the lateral average over the magnetization directions.

We give here the cross section in the case where the fluctuations are not interface ones, but bulk density fluctuations within the layers separated by flat interfaces. This is, for example, the case of magnetic domains with straight and vertical domain walls. This does not restrict the generality of the physics involved but the equations obtained are less lengthy than in the interface case. ${ }^{47,51-53}$ In Sec. II E the result in the small roughness amplitude approximation, extended to the scattering of polarized neutrons, is presented.

In the case of bulk density fluctuations, the interaction potential within each layer $l$ has a dependence only along the lateral coordinate $\boldsymbol{\rho}$ within the plane of the layers and the decomposition of the potential takes the following form:

$$
\hat{V}_{l}(\boldsymbol{\rho})=\hat{V}_{l}+\hat{\mathcal{V}}_{l}(\boldsymbol{\rho}),
$$

where $\hat{V}_{l}$ is the reference potential and $\hat{\mathcal{V}}_{l}(\boldsymbol{\rho})$ is the residual one treated as a perturbation such that $\left\langle\hat{\mathcal{V}}_{l}\right\rangle_{\boldsymbol{\rho}}=0$, where $\langle\cdot\rangle_{\boldsymbol{\rho}}$ stands for the average over the lateral coordinate $\boldsymbol{\rho}$ (i.e., over the sample surface). This decomposition is schematically represented in Fig. 2. The reference potential gives rise to the specular reflectivity and is written like in the preceding subsection and the residual one leads to scattering and writes $\hat{\mathcal{V}}_{l}(\boldsymbol{\rho})=2 \pi \hbar^{2} / m\left[\widetilde{\boldsymbol{\rho}}_{l}^{N}(\boldsymbol{\rho}) \cdot \hat{1}+\widetilde{\boldsymbol{\rho}}_{l}^{M}(\boldsymbol{\rho}) \cdot \hat{\boldsymbol{\sigma}} \widetilde{\mathbf{b}}_{l}\right]$, where $\widetilde{\boldsymbol{\rho}}_{l}^{N}(\boldsymbol{\rho})$ describes the lateral nuclear scattering length density fluctuations in layer 1, $\widetilde{\rho}_{l}^{M}(\boldsymbol{\rho})$ describes the lateral magnetic scattering length density fluctuations, and the unit vector $\widetilde{\mathbf{b}}_{l}$ the 
direction along which the magnetic field fluctuations in layer $l$ are oriented.

Within a coherence volume of the beam, the scattering amplitude of neutrons incident with wave vector $\mathbf{k}_{i}=\boldsymbol{\kappa}_{i}$ $+p_{i 0} \cdot \mathbf{e}_{z}$ and detected with wave vector $\mathbf{k}_{f}=\boldsymbol{\kappa}_{f}+p_{f 0} \cdot \mathbf{e}_{z}\left(\boldsymbol{\kappa}_{i}\right.$ and $\boldsymbol{\kappa}_{f}$ being the components of the wave vectors in the film plane) then writes ${ }^{30,31}$

$$
\mathcal{F}\left(\mathbf{k}_{f}, \mathbf{k}_{i}\right)=-\frac{m}{2 \pi \hbar^{2}} \sum_{l} \int d \mathbf{r}\left\langle\psi_{f l}\left(\mathbf{k}_{f}, \mathbf{r}\right)\left|\hat{\mathcal{V}}_{l}(\boldsymbol{\rho})\right| \psi_{i l}\left(\mathbf{k}_{i}, \mathbf{r}\right)\right\rangle,
$$

where the bra and the ket in that equation are solutions of the Schrödinger equation for the reference potential inside layer $l$. The integral sign represents an integration over the intersection of the considered beam coherence volume and the volume of layer $l$. This scattering amplitude looks very much like a scattering amplitude within the Born approximation. However, contrarily to the Born approximation in which the potential is taken between a bra and a ket describing plane waves far before and far after interaction with the sample the grazing incidence scattering amplitude within the DWBA is obtained by taking the residual potential between a bra and a ket that describe waves that are distorted by the reference potential. It describes the process of an incident plane wave impinging on the sample surface, that is distorted by the reference potential into the sample, then scattered by a fluctuation into another solution of the Schrödinger equation for the reference potential, and leaving the sample in the form of another plane wave. The strong dynamical effects observed at $\alpha_{i}$ or $\alpha_{f}$ close to a critical angle of total reflection are explicitly taken into account in the bra and the ket within the formalism of reflectance and transmittance described in Sec. II B. Note also that in the case of a multi-bilayer or a supermirror for which the reflection coefficients at the multilayer Bragg positions can have large values, the scattering cross section calculated within the DWBA differs substantially from the one calculated within the Born approximation.

Also, the bra and the ket in Eq. (7) assume different asymptotic conditions. The ket assumes an incident plane wave that approaches the sample from the source of radiation, whereas the bra assumes an incident plane wave that approaches the sample from the side of the detector. Within each layer $l$ the reference potential is constant, i.e., it does not depend on $\mathbf{r}$. The ket in layer $l$ then writes

$$
\left|\psi_{i l}\left(\mathbf{k}_{i}, \mathbf{r}\right)\right\rangle=e^{i \boldsymbol{\kappa}_{i} \boldsymbol{\rho}} \cdot \hat{S}_{i l}(z) \cdot\left|\psi_{i 0}\left(\mathbf{k}_{i}, 0\right)\right\rangle
$$

with the propagator in layer $l$ :

$$
\hat{S}_{i l}(z)=e^{i \hat{p}_{i l}\left(z-z_{l-1}\right)} \hat{t}_{i l}+e^{-i \hat{p}_{i l}\left(z-z_{l-1}\right)} \hat{r}_{i l}
$$

and the bra writes

$$
\left\langle\psi_{f l}\left(\mathbf{k}_{f}, \mathbf{r}\right)\right|=\left\langle\psi_{f 0}\left(\mathbf{k}_{f}, 0\right)\right| \cdot \hat{S}_{f l}(z) \cdot e^{-i \boldsymbol{\kappa}_{f} \boldsymbol{\rho}},
$$

where $\boldsymbol{\kappa}_{f}$ is the in-plane (conserving) component of $\mathbf{k}_{f}$ and with the associated propagator:

$$
\hat{S}_{f l}(z)=\hat{t}_{f l} e^{i \hat{p}_{f l}\left(z-z_{l-1}\right)}+\hat{r}_{f l} e^{-i \hat{p}_{f l}\left(z-z_{l-1}\right)} .
$$

The reflection $\hat{r}_{i l}$ and transmission $\hat{t}_{i l}$ amplitude operators used to build the propagator for the ket in Eq. (8) can be calculated from Eq. (4). The reflection $\hat{r}_{f l}$ and transmission $\hat{t}_{f l}$ amplitude operators in layer $l$ used to build the propagator for the bra in Eq. (10) can be deduced in a similar manner as in the preceding subsection, by writing the continuity relations at the interfaces. $\hat{p}_{f l}=\sqrt{p_{f 0}^{2} \hat{1}-\hat{p}_{c, l}^{2}}$ is the component of the scattered wave vector in layer $l$ perpendicular to the sample surface, with $p_{f 0}=k_{f} \sin \left(\alpha_{f}\right)$ the component of $\mathbf{k}_{f}$ in vacuum perpendicular to the sample surface. The eigenvaluesp $p_{f l}^{ \pm}$of $\hat{p}_{f l}$ have the same functional form as the ones of $\hat{p}_{i l}$ in the previous subsection, and $\hat{p}_{f l}$ can also be written as a linear combination of the unit matrix and the Pauli matrices as for $\hat{p}_{i l}$ in Eq. (3).

The scattering amplitude can therefore be rewritten in the following way:

$$
\mathcal{F}\left(\mathbf{k}_{f}, \mathbf{k}_{i}\right)=\left\langle\psi_{f 0}\left(\mathbf{k}_{f}, 0\right)\left|\hat{F}_{f i}\right| \psi_{i 0}\left(\mathbf{k}_{i}, 0\right)\right\rangle
$$

with the scattering amplitude operator:

$$
\hat{F}_{f i}=\sum_{l} \int d z \hat{S}_{f l}(z) \cdot \hat{F}_{l}\left(\mathbf{Q}_{\|}\right) \cdot \hat{S}_{i l}(z)
$$

and the lateral Fourier transform of the residual potential:

$$
\hat{F}_{l}\left(\mathbf{Q}_{\|}\right)=-\frac{m}{2 \pi \hbar^{2}} \int d \boldsymbol{\rho} e^{-i \mathbf{Q}_{\|} \cdot \boldsymbol{\rho}} \hat{\mathcal{V}}_{l}(\boldsymbol{\rho}), \quad \mathbf{Q}_{\|}=\boldsymbol{\kappa}_{f}-\boldsymbol{\kappa}_{i} .
$$

The integral signs in Eqs. (13) and (14) represent an integration over the height of layer $l$ and an integration over the intersection of the considered beam coherence volume and the surface of the sample, respectively. Therefore, similarly to the calculation of the scattering amplitude within the Born approximation, the calculation of the scattering amplitude within the DWBA includes the lateral Fourier transform of the residual potential. The other terms entering in Eq. (13) depend only on the reference potential and not on the fluctuations.

Inserting formulas (9) and (11) for the propagation operators into Eq. (13), the scattering amplitude operator can be rewritten in the following way:

$$
\hat{F}_{f i}=\sum_{l}\left(\hat{t}_{f l} \hat{F}_{f i l}^{t t} \hat{t}_{i l}+\hat{t}_{f l} \hat{F}_{f i l}^{t r} \hat{r}_{i l}+\hat{r}_{f l} \hat{F}_{f i l}^{r t} \hat{t}_{i l}+\hat{r}_{f l} \hat{F}_{f i l}^{r r} \hat{r}_{i l}\right) .
$$

The terms on the right-hand side of Eq. (15) have a clear physical meaning; they describe several processes contributing to the scattered wave. For instance, the term $\hat{t}_{f l} \hat{F}_{\text {fil }}^{t t} \hat{t}_{i l}$ describes the scattering from the transmitted wave in layer $l$ with amplitude $\hat{t}_{i l}$ into the transmitted wave with amplitude $\hat{t}_{f l}$. Similarly, the process corresponding to the term $\hat{t}_{f l} \hat{F}_{f i l}^{t r} \hat{r}_{i l}$ is a scattering from the reflected wave in layer $l$ with amplitude $\hat{r}_{i l}$ into the transmitted wave with amplitude $\hat{t}_{f l}$. The physical meaning of the two other terms is similar.

The exponential operators entering in the formula for the propagators in Eqs. (9) and (11) are functions of linear com- 
binations of the Pauli matrices and the unit matrices. Therefore, in the same way as in the previous subsection for the operator component of the wave vector perpendicular to the sample surface [Eq. (3)], they can be decomposed into other linear combinations of the Pauli matrices and the unit matrix. For example, for the first exponential operator in Eq. (9), one has

$$
\begin{aligned}
e^{i \hat{p}_{i l}\left(z-z_{l-1}\right)}= & \frac{1}{2}\left[\left(e^{i p_{i l}^{+}\left(z-z_{l-1}\right)}+e^{i p_{i l}^{-}\left(z-z_{l-1}\right)}\right) \hat{1}\right. \\
& \left.+\left(e^{i i_{i l}^{+}\left(z-z_{l-1}\right)}-e^{i p_{i l}^{-}\left(z-z_{l-1}\right)}\right) \hat{\boldsymbol{\sigma}} \mathbf{b}_{l}\right] .
\end{aligned}
$$

Inserting those decompositions into the propagators and performing the integration over $z$ in Eq. (13), the partial scattering amplitude operators $\hat{F}_{f i l}^{\alpha \beta}(\alpha, \beta=t$ or $r)$ write

$$
\hat{F}_{f i l}^{\alpha \beta}=\frac{1}{4} \sum_{\mu \nu} g_{f i l}^{\alpha \beta \mu \nu}\left(1+\mu \cdot \hat{\boldsymbol{\sigma}} \mathbf{b}_{l}\right) \hat{F}_{l}\left(\mathbf{Q}_{\|}\right)\left(1+\nu \cdot \hat{\boldsymbol{\sigma}} \mathbf{b}_{l}\right),
$$

where $\mu= \pm, \nu= \pm$ represent the spin projections of the incident and final neutron waves along a quantization axis, respectively. The Laue functions $g_{f i l}^{\alpha \beta \mu \nu}$ are obtained from the integration over the layer thickness $d_{l}$ :

$$
g_{f i l}^{\alpha \beta \mu \nu}=\frac{\exp \left(i q_{f i l}^{\alpha \beta \mu \nu} d_{l}\right)-1}{i q_{f i l}^{\alpha \beta \mu \nu}}
$$

with $q_{f i l}^{\alpha \beta \mu \nu}$ the wave vector transfers perpendicular to the film surface when only the reference Hamiltonian is considered: $q_{f i l}^{t t \mu \nu}=p_{f l}^{\mu}+p_{i l}^{\nu}, q_{f i l}^{t r \mu \nu}=p_{f l}^{\mu}-p_{i l}^{\nu}, q_{f i l}^{r t \mu \nu}=-p_{f l}^{\mu}+p_{i l}^{\nu}$, and $q_{f i l}^{r r \mu \nu}$ $=-p_{f l}^{\mu}-p_{i l}^{\nu}$.

The Laue functions $g_{f i l}^{t t \mu \nu}$ describe the different spin-flip and non-spin-flip processes that can occur in layer $l$ when the transmitted wave with amplitude $\hat{t}_{i l}$ is scattered into the transmitted wave with amplitude $\hat{t}_{f l} . g_{\text {fil }}^{t t++}$ and $g_{\text {fil }}^{t t--}$ correspond to non-spin-flip processes and show sharp maxima at $p_{f l}^{+}+p_{i l}^{+}=0$ and $p_{f l}^{-}+p_{i l}^{-}=0$, respectively. The Laue functions $g_{\text {fil }}^{t+-}$ and $g_{\text {fil }}^{t t-+}$ correspond to spin-flip processes and show sharp maxima at $p_{f l}^{+}+p_{i l}^{-}=0$ and $p_{f l}^{-}+p_{i l}^{+}=0$, respectively. The physical meaning of the other Laue functions is similar. Therefore, inside each layer $1, \hat{F}_{f i}$ decomposes into $4 \times 4$ terms, representing the sixteen types of scattering process that can occur.

After having discussed the Laue functions $g_{\text {fil }}^{\alpha \beta \mu \nu}$, we now focus on the term $\hat{F}_{l}\left(\mathbf{Q}_{\|}\right)$in Eq. (17), which is the lateral Fourier transform of the fluctuations in layer 1. It decomposes into

$$
\hat{F}_{l}\left(\mathbf{Q}_{\|}\right)=\rho_{l}^{N} \hat{1} F_{l}^{N}\left(\mathbf{Q}_{\|}\right)+\rho_{l}^{M} \hat{\boldsymbol{\sigma}} \tilde{\mathbf{b}}_{l \perp} F_{l}^{M}\left(\mathbf{Q}_{\|}\right),
$$

where

$$
\begin{aligned}
& F_{l}^{N}\left(\mathbf{Q}_{\|}\right)=-\frac{m}{2 \pi \hbar^{2}} \int d \boldsymbol{\rho} e^{-i \mathbf{Q}_{\|} \boldsymbol{\rho}} \widetilde{\boldsymbol{\rho}}_{l}^{N}(\boldsymbol{\rho}) / \rho_{l}^{N}, \\
& F_{l}^{M}\left(\mathbf{Q}_{\|}\right)=-\frac{m}{2 \pi \hbar^{2}} \int d \boldsymbol{\rho} e^{-i \mathbf{Q}_{\|} \boldsymbol{\rho} \widetilde{\boldsymbol{\rho}}_{l}^{M}(\boldsymbol{\rho}) / \rho_{l}^{M}}
\end{aligned}
$$

are the lateral Fourier transforms of the nuclear and magnetic scattering length density fluctuations (relative to their later- ally averaged counterparts), respectively, and $\tilde{\mathbf{b}}_{l \perp}$ is the component of $\widetilde{\mathbf{b}}_{l}$ perpendicular to $\mathbf{Q}$. In other words, $\widetilde{\mathbf{b}}_{l \perp}$ is the Halpern-Johnson vector for magnetic scattering of neutrons $^{54,55}$ defined by $\widetilde{\mathbf{b}}_{l \perp}=\widetilde{\mathbf{b}}_{l}-\left(\tilde{\mathbf{b}}_{l} \cdot \hat{\mathbf{Q}}\right) \cdot \hat{\mathbf{Q}}$, where $\hat{\mathbf{Q}}$ is the unit vector parallel to $\mathbf{Q}$.

Finally, the scattering cross section is obtained by taking the modulus squared of the scattering amplitude in Eq. (12). The result has also to be averaged over the different states $\left|\psi_{i 0}\left(\mathbf{k}_{i}, 0\right)\right\rangle$ and $\left\langle\psi_{f 0}\left(\mathbf{k}_{f}, 0\right)\right|$ prepared by the polarizer and accepted by the analyzer. In the same way as for the specular reflectivity, the scattering cross section can therefore be written

$$
\frac{d \sigma}{d \Omega}\left(p_{i 0}, p_{f 0}, \mathbf{Q}_{\|}\right)=\left\langle\operatorname{Tr}\left(\hat{\rho}_{f} \hat{F}_{f i} \hat{\rho}_{i} \hat{F}_{f i}^{+}\right)\right\rangle,
$$

where the added bracket sign $\langle\cdot\rangle$ stands for a sum over all the coherence surfaces illuminated by the beam.

By inserting Eq. (19) into Eqs. (17), (15), and (21), one can show that the total cross section can be decomposed into several parts, involving terms of the type $F_{l}^{N}\left(\mathbf{Q}_{\|}\right) \cdot F_{l^{\prime}}^{N *}\left(\mathbf{Q}_{\|}\right), \quad\left(\hat{\boldsymbol{\sigma}} \tilde{\mathbf{b}}_{l \perp}\right) F_{l}^{M}\left(\mathbf{Q}_{\|}\right) \cdot\left(\hat{\boldsymbol{\sigma}} \tilde{\mathbf{b}}_{l^{\prime} \perp}\right) F_{l^{\prime}}^{M *}\left(\mathbf{Q}_{\|}\right), \quad$ and $F_{l}^{N}\left(\mathbf{Q}_{\|}\right) \cdot\left(\hat{\boldsymbol{\sigma}} \widetilde{\mathbf{b}}_{l^{\prime} \perp}\right) F_{l^{\prime}}^{M *}\left(\mathbf{Q}_{\|}\right)$, products of two Fourier transforms of the lateral fluctuations, and therefore equal to the Fourier transforms of the lateral pair correlations between the nuclear-nuclear, magnetic-magnetic and nuclear-magnetic density fluctuations respectively. Therefore, similarly to the Born approximation, the calculation of the scattering cross section within the DWBA can be reduced to the modeling of pair correlation functions of the fluctuations. The other terms are independent of the fluctuations and depend only on the reference potential. They influence drastically the scattering cross section at angles $\alpha_{i}$ and $\alpha_{f}$ close to total reflection or at angles for which the average multilayer structure (i.e., the reference potential) induces strong Bragg peaks.

\section{Application to bulk lateral correlations of the spin misalignment}

In order to describe the measured data, we assume a model with the mean magnetizations either parallel or antiparallel to the external field $\mu_{0} \mathbf{H}$ applied along the $y$ axis of Fig. 1. We also take this axis as the quantization axis of the neutron spin. Lateral correlations in layer $l$ are described by neighboring regions of constant magnetization with the modulus equal to the saturation magnetization, of average size $2 \xi_{l}^{M}$, in which the spins are misaligned with respect to the applied field $\mu_{0} \mathbf{H}$, making alternatively angles $\varphi_{l}$ and $-\varphi_{l}$ with respect to the field axis. $\varphi_{l}$ is not constant over the whole surface of the sample; it varies randomly from region to region around a mean value $\Phi_{l}$ with a Gaussian distribution of rms width equal to $\omega_{l}$. A schematic representation of the model is given in Fig. 3. Note that random fluctuations of the spin misalignment were already introduced in order to describe small angle scattering data from soft nanocrystalline bulk ferromagnets. ${ }^{56-58}$

The average magnetic scattering length density in layer $l$, leading to non-spin-flip specular reflectivity is given by $\rho_{l}^{M}$ 


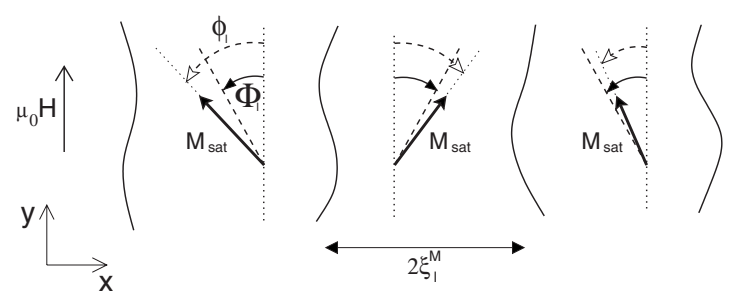

FIG. 3. Model of lateral magnetic correlations. $\varphi_{l}$, the angle defining the directions of the magnetization with respect to the applied magnetic field $\mu_{0} \mathbf{H}$ inside the laterally correlated regions, varies randomly from one region to another around the mean value $\Phi_{l}$ following a Gaussian distribution of rms width $\omega_{l}$.

$=\rho_{l}^{M \text { sat }} \cos \left(\Phi_{l}\right)$ where $\rho_{l}^{M \text { sat }}$ is the magnetic scattering length density in layer $l$ at magnetic saturation. Two types of scattering processes are expected. If $\Phi_{l}$ is not equal to $0^{\circ}$ or $180^{\circ}$, then lateral fluctuations of the $x$ component of the magnetization (transverse fluctuations) are expected, which lead to spin-flip off-specular scattering, as those fluctuations are oriented perpendicular to the quantization axis of the neutrons $\left(\tilde{\mathbf{b}}_{l} \perp \mu_{0} \mathbf{H}\right)$. If $\omega_{l}$ is not equal to zero, the $y$ component of the magnetizations will also fluctuate (longitudinal fluctuations), leading to non-spin-flip scattering, as those fluctuations are oriented parallel to the quantization axis $\left(\tilde{\mathbf{b}}_{l} \| \mu_{0} \mathbf{H}\right)$.

Considering homogeneous boxes of lateral size $2 \xi_{l}^{M}$ in layer 1, smaller than the lateral coherence length of the beam, the Fourier transforms of the pair correlation functions $\mathbf{e}_{x} F_{l}^{M}\left(\mathbf{Q}_{\|}\right) \cdot \mathbf{e}_{x} F_{l^{\prime}}^{M *}\left(\mathbf{Q}_{\|}\right)$and $\mathbf{e}_{y} F_{l}^{M}\left(\mathbf{Q}_{\|}\right) \cdot \mathbf{e}_{y} F_{l^{\prime}}^{M *}\left(\mathbf{Q}_{\|}\right)$for transverse and longitudinal fluctuations, respectively, can be calculated. ${ }^{59}$ After summation over the surfaces of coherence and integration over the $y$-component $Q_{y}$ of $\mathbf{Q}$ which is not resolved in this experiment, they become

$$
\begin{aligned}
& \overline{\left\langle\mathbf{e}_{x} F_{l}^{M} \cdot \mathbf{e}_{x} F_{l^{\prime}}^{\left.M^{*}\right\rangle^{y}}\right.}\left(Q_{x}\right) \frac{\mathcal{N}_{0} \cdot \mathcal{S}_{\mathrm{coh}} \cdot S_{l l^{\prime}}^{M}}{2} \cdot\left[\frac{\xi_{l}^{M}}{1+\left(Q_{x} \cdot \xi_{l}^{M}\right)^{2}}+\frac{\xi_{l^{\prime}}^{M}}{1+\left(Q_{x} \cdot \xi_{l^{\prime}}^{M}\right)^{2}}\right], \\
& \overline{\left\langle\mathbf{e}_{y} F_{l}^{M} \cdot \mathbf{e}_{y} F_{l^{\prime}}^{M *}\right\rangle^{y}}\left(Q_{x}\right) \\
&=\frac{\mathcal{N}_{0} \cdot \mathcal{S}_{\mathrm{coh}} \cdot C_{l l^{\prime}}^{M}}{2} \cdot\left[\frac{\xi_{l}^{M}}{1+\left(Q_{x} \cdot \xi_{l}^{M}\right)^{2}}+\frac{\xi_{l^{\prime}}^{M}}{1+\left(Q_{x} \cdot \xi_{l^{\prime}}^{M}\right)^{2}}\right],
\end{aligned}
$$

where $\mathcal{S}_{\text {coh }}$ is the surface of coherent illumination by the beam and $\mathcal{N}_{0}$ is the number of such surfaces that cover the whole illuminated area. The Fourier transforms of the pair correlation functions have a Lorentzian shape in $Q_{x} . \quad S_{l l^{\prime}}^{M}=\left\langle\sin \left(\varphi_{l}\right) \cdot \sin \left(\varphi_{l^{\prime}}\right)\right\rangle \quad$ and $\quad C_{l l^{\prime}}^{M}=\left\langle\cos \left(\varphi_{l}\right) \cdot \cos \left(\varphi_{l^{\prime}}\right)\right\rangle$ $-\left\langle\cos \left(\varphi_{l}\right)\right\rangle^{2} \delta_{l l^{\prime}}$ are given by the amplitudes of fluctuation of the magnetizations perpendicular and parallel to the field axis, respectively. It can easily be shown that $S_{l l}^{M}=\frac{1}{2}\left[1-\cos \left(2 \Phi_{l}\right) \cdot e^{-2 \omega_{l}^{2}}\right]$ and $C_{l l}^{M}=\frac{1}{2}\left[1-\cos \left(2 \Phi_{l}\right) \cdot e^{-\omega_{l}^{2}}\right][1$ $\left.-e^{-\omega_{l}^{2}}\right]$ for the autocorrelations within layer 1 .

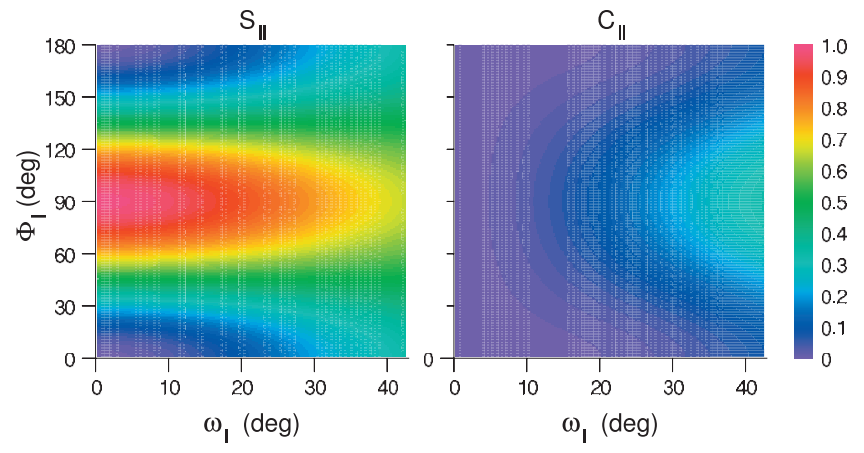

FIG. 4. (Color) Spin-spin autocorrelation functions in layer $l$ for the spin components perpendicular $\left(S_{l l}\right)$ and parallel $\left(C_{l l}\right)$ to the applied field $\mu_{0} \mathbf{H}$ as a function of the parameter $\Phi_{l}$, angle giving the average directions of the magnetizations in the spin correlated regions, and the rms amplitude $\omega_{l}$ of the fluctuations around the average directions.

Those factors are plotted in Fig. 4. At $\Phi_{l}=0$ and $\omega_{l}=0$, i.e., all magnetizations along the field and no fluctuation, $S_{l l}^{M}=C_{l l}^{M}=0$. When the amplitude of the fluctuations $\left(\omega_{l}\right)$ around the mean value $\Phi_{l}=0$ increases, both off-specular scattering from perpendicular and off-specular scattering from parallel fluctuations increase, $S_{l l}^{M}$ increasing faster than $C_{l l}^{M}$. At $\omega_{l}=0$, the scattering from perpendicular fluctuations increases steeply with $\Phi_{l}$ and $C_{l l}^{M}$ stays equal to zero. The scattering from fluctuations perpendicular to the applied field appears in the SF channels and the scattering from parallel fluctuations in the NSF channels. Therefore, from intensity comparison between the two types of channels, it is possible to determine uniquely the $\Phi_{l}$ and $\omega_{l}$ parameters.

To take into account cross-correlations between two layers $l$ and $l '$ one can write, as a simplest model, $S_{l l^{\prime}}^{M}$ $=\sqrt{S_{l l}^{M} S_{l^{\prime} l^{\prime}}^{M}} \cdot e^{-\left|z l^{-}-z_{l^{\prime}}\right| \xi_{\perp}^{M}}$, with $\xi_{\perp}^{M}$ taken independent on the pair of layers considered, and $C_{l l^{\prime}}^{M}=0$. The larger the vertical correlation length $\xi_{\perp}^{M}$, the stronger the cross-correlations.

\section{E. Scattering at interfaces in the small $Q_{\perp} \cdot \sigma$ limit}

The first full derivation of the scattering cross section from the air/substrate random interface within the DWBA was given by Sinha et al., ${ }^{47}$ and has been latter extended to random interfaces in multilayers. ${ }^{51-53,60}$ More recently a cross section for the scattering of soft $\mathrm{x}$ rays by magnetically rough interfaces has been presented. ${ }^{61,62}$ Here we give the final result in the small $Q_{\perp} \cdot \sigma$ limit, ${ }^{51,53}$ that we extend to polarized neutron scattering from interfaces between magnetic layers. In the following, we make the particular assumption that the magnetic profile of the interfaces follows exactly the nuclear profile and that the magnetizations close to the interfaces are equal to the magnetizations in the bulk of the layer (i.e., no magnetic roughness). Note that in general interfaces will appear "smoother" for magnetic scattering as compared to nuclear scattering, since the first is due to the magnetic induction field, while the later is due to a very local scattering process at the nuclei.

We assume that the interface heights are Gaussian random variables of the lateral coordinate and that the interface $l$ on 
top of layer $l$ fluctuates in height with a rms amplitude that saturates to a value $\sigma_{l}$ at lateral distances well above a cutoff length $\xi_{l}^{R}{ }^{47}$ In the small $Q_{\perp} \sigma$ approximation, the scattering cross section can be written in the same way as in Eq. (21) but with the partial scattering amplitude operators [Eq. (17)] replaced by the following operator:

$$
\hat{F}_{l}^{\alpha \beta}=\frac{1}{4} \sum_{\mu \nu} e^{-1 / 2\left(q_{f i l}^{\alpha \beta \mu \nu} \sigma_{l}\right)^{2}}\left(1+\mu \cdot \hat{\boldsymbol{\sigma}} \mathbf{b}_{l}\right) \hat{F}_{l}\left(\mathbf{Q}_{\|}\right)\left(1+\nu \cdot \hat{\boldsymbol{\sigma}} \mathbf{b}_{l}\right) .
$$

In this equation, the Laue functions in Eq. (18), resulting from the $z$ integration of residual potentials that do not depend on $z$ within a layer, are replaced by Debye-Waller factors, resulting from the integration of potential differences with $z$ extensions fluctuating randomly with the lateral coordinate around the average heights of the interfaces

$$
\hat{F}_{l}\left(\mathbf{Q}_{\|}\right)=\left[\left(\rho_{l}^{N} \hat{1}+\rho_{l}^{M} \hat{\boldsymbol{\sigma}} \mathbf{b}_{l \perp}\right)-\left(\rho_{l-1}^{N} \hat{1}+\rho_{l-1}^{M} \hat{\boldsymbol{\sigma}} \mathbf{b}_{l-1 \perp}\right)\right] F_{l}^{N}\left(\mathbf{Q}_{\|}\right),
$$

where $F_{l}^{N}\left(\mathbf{Q}_{\|}\right)$is the lateral Fourier transform of the height of interface $l$ and $\mathbf{b}_{l \perp}=\mathbf{b}_{l}-\left(\mathbf{b}_{l} \cdot \hat{\mathbf{Q}}\right) \cdot \hat{\mathbf{Q}}$. Note that the contrasts in both nuclear and magnetic scattering length densities at the interface enter.

For the computation of the scattering cross section in Eq. (21), we are left in calculating the product $F_{l}^{N}\left(\mathbf{Q}_{\|}\right) \cdot F_{l^{\prime}}^{N *}\left(\mathbf{Q}_{\|}\right)$, which is equal to the Fourier transform of the height-height auto- $\left(l=l^{\prime}\right)$ or cross- $\left(l \neq l^{\prime}\right)$ correlation functions between interfaces $l$ and $l$ '. Assuming self-affine rough interfaces with a cutoff ${ }^{47}$ length $\xi_{l}^{R}$, root mean squared (rms) amplitudes $\sigma_{l}$, and the same Hurst parameter $h=0.5$ for all interfaces, the Fourier transforms of the height-height correlation functions can be written, after integration over $Q_{y}$ :

$$
\begin{aligned}
\overline{\left\langle F_{l}^{N} \cdot F_{l^{\prime}}^{N *}\right\rangle^{y}}\left(Q_{x}\right) \\
=\frac{\mathcal{N}_{0} \cdot \mathcal{S}_{\mathrm{coh}} \cdot C_{l l^{\prime}}^{R}}{2} \cdot\left[\frac{\xi_{l}^{R}}{1+\left(Q_{x} \cdot \xi_{l}^{R}\right)^{2}}+\frac{\xi_{l^{\prime}}^{R}}{1+\left(Q_{x} \cdot \xi_{l^{\prime}}^{R}\right)^{2}}\right]
\end{aligned}
$$

with $C_{l l^{\prime}}^{R}=\sigma_{l} \cdot \sigma_{l^{\prime}} \cdot e^{-\left|z_{l}-z_{l^{\prime}}\right|} \mid \xi_{\perp}^{R}$ where $\xi_{\perp}^{R}$, taken independent on the pair of interfaces considered, is a measure of the conformity of the interfaces. ${ }^{63}$ When the vertical correlation length $\xi_{\perp}^{R}$ is large with respect to the distance between two interfaces, the lateral height profiles of those interfaces evolve approximately in phase. The Fourier transforms of the height-height correlation functions have a Lorentzian shape in $Q_{x}$.

\section{SAMPLE}

Polarizing supermirrors are commonly used for the polarization of cold or thermal neutron beams. They consist of a series of alternating ferromagnetic and nonmagnetic layers with a gradient in the layer thickness. In a remanent polarizing supermirror, a macroscopic uniaxial anisotropy allows one to keep a remanent magnetization after the magnetizing
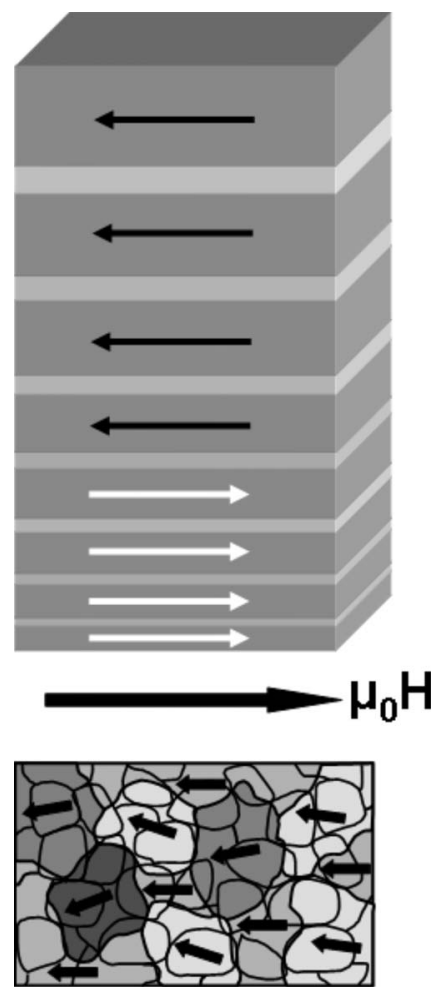

FIG. 5. Sample consists of $100 \mathrm{Fe}_{50} \mathrm{Co}_{48} \mathrm{~V}_{2}$ layers separated by $100 \mathrm{TiN}_{x}$ layers. The $\mathrm{Fe}_{50} \mathrm{Co}_{48} \mathrm{~V}_{2} / \mathrm{TiN}_{x}$ bilayer thickness increases continuously from the bottom to the top of the structure. See Sec. III for details. The bottommost ferromagnetic layers have lower coercivities than the topmost. Below a certain grain size, exchange coupling leads to the formation of regions larger than the grains in which the magnetization is approximately uniform.

field is switched off or even slightly reversed. In this state, the supermirror reflects neutrons with the spin opposite to the guide field (i.e., "-" neutrons). This feature can be used to build a polarized neutron instrument without the use of a spin flipper. ${ }^{34}$

The present sample under investigation, commercially available from SwissNeutronics, ${ }^{34}$ contains $100 \mathrm{Fe}_{50} \mathrm{Co}_{48} \mathrm{~V}_{2}$ layers separated by $100 \mathrm{TiN}_{x}$ layers. A Schematic of the structure is represented in Fig. 5 . $\mathrm{Fe}_{50} \mathrm{Co}_{48} \mathrm{~V}_{2}$ is chosen for its large scattering length density for " + " neutrons, $\rho_{\mathrm{FeCoV}}^{+}$ $=\rho_{\mathrm{FeCoV}}^{N}+\rho_{\mathrm{FeCoV}}^{M}$, leading to a relatively large critical angle of total reflection $\left(\alpha_{c}^{+}=1.8 \mathrm{mrad} / \AA\right)$, whereas it does not total reflect "-" neutrons, because $\rho_{\mathrm{FeCoV}}^{-}=\rho_{\mathrm{FeCoV}}^{N}-\rho_{\mathrm{FeCoV}}^{M}<0$. In order to increase the angular range of good reflectivity above this critical angle, up to $m=2.5$ times the critical angle of $\mathrm{Ni}$, the $\mathrm{FeCoV} / \mathrm{TiN}$ bilayer thickness is increased continuously from the substrate to the top, nominally from 11.6 to $82.6 \mathrm{~nm}$. The variation is not linear. The thickness increases very slowly for the first 70 bilayersand then steeper for the remaining bilayers. ${ }^{64} \mathrm{An}$ important point for the later discussion is to notice that, although the $\mathrm{FeCoV}$ layers are all thicker than their neighboring $\mathrm{TiN}_{x}$, the thicknesses of the first $70 \mathrm{FeCoV}$ layers differ by less than $15 \%$ of the thicknesses of their first nearest neighbor $\operatorname{TiN}_{x}$ layers, i.e., the first $70 \mathrm{FeCoV} / \mathrm{TiN}_{x}$ bilayers consist of layers having almost the same thickness. 
The content of nitrogen in $\operatorname{TiN}_{x}$ was adjusted by tuning the partial pressure of $\mathrm{N}_{2}$ during the reactive sputtering process such that the contrast $\rho_{\mathrm{FeCoV}}^{-}-\rho_{\mathrm{TiN}_{\mathrm{r}}}^{N}$ vanishes. In order to absorb the "-" neutrons that are transmitted by the device, a $45 \mathrm{~nm}$ thick Gd layer is inserted between the supermirror structure and the borated glass substrate.

The supermirror was produced using sputtering conditions leading to an anisotropic stress distribution within the plane of the layers. ${ }^{34}$ Due to magnetoelastic coupling, a well defined easy axis of magnetization is induced, coherent over the whole sample surface, providing an increased remanence. The remanence is such that the magnetization distribution is almost not affected by switching off the magnetizing field, or even by slightly reversing it. We will show later in this article that this is not exactly true.

Structural and magnetic investigations have already been performed on such supermirrors or on multilayers composed of the same materials and substrate. From macroscopic magnetometry and polarized neutron reflectometry, it has been shown that the magnetic coercivity of the $\mathrm{FeCoV}$ layers increases with thickness. ${ }^{34,39}$ At the same time, an increase of the grain size with the layer thickness was reported,${ }^{39}$ leading to a correlation between grain size and amplitude of the coercive field. This correlation was explained using the random anisotropy model. ${ }^{35}$ The same $\mathrm{FeCoV}$ ferromagnet is also implemented in multilayer structures for applications in spintronics. ${ }^{37,38,65-68}$

In Sec. V A, a detailed investigation of the remagnetization process by polarized neutron reflectivity with polarization analysis is presented, confirming the previous investigations. A first contribution to this study has already been published elsewhere. ${ }^{69}$ In Sec. V B, a depth-resolved investigation of the in-plane structural and magnetic correlations is performed by off-specular scattering with polarization analysis, giving more detailed information on the mechanism of coercivity in this system.

\section{EXPERIMENT}

\section{A. Reflectometer}

Reflectivity and off-specular scattering with polarization analysis were measured on the HADAS reflectometer located at the FRJ-2 reactor in Jülich. ${ }^{70}$ The wavelength was $\lambda$ $=0.452 \mathrm{~nm}$ with a wavelength spread of $1.1 \%$ (FWHM). In order to define with good enough precision the angle of incidence $\alpha_{i}$, the incident beam was collimated by two slits parallel to the $y$ axis of Fig. 1, $1 \mathrm{~mm}$ in width, located 1450 and $470 \mathrm{~mm}$ before the $60 \mathrm{~mm}$ wide sample. The data were collected on a $80 \mathrm{~mm}$ diameter 2D position sensitive detector (PSD) and integrated over the angle $\varphi$ in Fig. 1.

After interaction with the sample, the neutrons are detected on the PSD, permitting the simultaneous detection of the reflected and off-specular scattered neutrons over a whole range $(\approx 53 \mathrm{mrad})$ of $\alpha_{f}$ angles. In order to obtain the $\left(\alpha_{i}, \alpha_{f}\right)$ maps presented in this article, two scans were performed, scanning $\alpha_{i}$ from 0 to $70 \mathrm{mrad}$ at two fixed values of $\alpha_{f}$ for the detector center, namely 19.2 and $59.3 \mathrm{mrad}$.

The polarization of the neutrons is obtained with the same supermirror as the one presented in the previous section. A beam of polarized neutrons in the $|+\rangle$ or $|-\rangle$ state can therefore be obtained without the use of a spin-flipper. After interaction with the sample, the projection of the neutron spin on the quantization axis can be reversed by the use of a Mezei-type spin flipper. The unique feature of this instrument lies in its polarization analyzer. ${ }^{71}$ It consists of a stack of magnetically saturated polarizing supermirrors almost parallel to the scattering plane and set up as a divergent collimator, allowing the simultaneous analysis of all the neutrons coming from an up to $15 \mathrm{~mm}$ high illuminated region of the sample and hitting the PSD. At each magnetic field, the intensities corresponding to the four spin-flip and non-spin-flip cross-sections $\left(I_{+-}, I_{-+}, I_{++}\right.$and $\left.I_{--}\right)$for reflectivity and offspecular scattering are acquired. A more detailed description of this reflectometer can be found on our website. ${ }^{72}$ By now the instrument has been transferred to the FRM-II reactor, where it will be operated in a slightly modified version as "TREFF" reflectometer.

\section{B. Scattering geometry}

To a given pair of $\alpha_{i}$ and $\alpha_{f}$ angles corresponds the components of the momentum transfer along and perpendicular to the sample surface (respectively $x$ and $z$ directions in Fig. 1) that are given by $Q_{x}=k \cdot\left[\cos \left(\alpha_{f}\right)-\cos \left(\alpha_{i}\right)\right]$ $\approx k \cdot\left(\alpha_{i}-\alpha_{f}\right) \cdot\left(\alpha_{i}+\alpha_{f}\right) / 2$ and $Q_{\perp}=k \cdot\left[\sin \left(\alpha_{f}\right)+\sin \left(\alpha_{i}\right)\right]$ $\approx k \cdot\left(\alpha_{i}+\alpha_{f}\right)$, respectively. The approximations are performed taking into account that the angles $\alpha_{i}$ and $\alpha_{f}$ are small. In a $\left(\alpha_{i}, \alpha_{f}\right)$ map, the intensities along the main diagonal $\left(\alpha_{i}=\alpha_{f}\right)$ represent a scan along the $Q_{\perp}$ axis at $Q_{x}=0$, which is called specular scan. Intensities recorded along a second diagonal, i.e., varying $\left(\alpha_{i}-\alpha_{f}\right)$ at constant $\left(\alpha_{i}+\alpha_{f}\right)$, are a rocking scan. Thus rocking scans are nearly $Q_{x}$ scans at constant $Q_{\perp}$ and lateral correlations are probed. We have chosen to represent the off-specular data in $\left(\alpha_{i}, \alpha_{f}\right)$ maps rather than $\left(Q_{x}, Q_{\perp}\right)$ maps, because the intensities are strongly influenced by the amplitudes of the reflection and transmission coefficients that are, themselves, solely determined by the angles.

\section{Data analysis}

The measurements of reflectivity and off-specular scattering shown in the next section at four different magnetic fields are representative of the full set of data measured at 18 fields along the remagnetization of the sample. They are presented as they were measured, i.e., no correction was applied. The effects of limited polarization efficiencies, illumination and resolution convolution are properly taken into account in the simulations.

The polarization efficiencies appearing in the density matrices in Eqs. (5) and (21) are determined from the specular intensities by adjusting the values of $\mathbf{P}_{i}$ and $\mathbf{P}_{f}$ in order to reproduce the spin-flip intensities. As will be emphasized in the next subsection, we assume that the mean magnetizations are all parallel or antiparallel to the guiding field of the neutrons and that, therefore, all the observed spin-flip specular signal is due to polarization inefficiencies. Let $P_{i}$ and $P_{f}$ be the coordinates of $\mathbf{P}_{i}$ and $\mathbf{P}_{f}$ along the quantization axis. We assume the same absolute values of $P_{i}$ and $P_{f}$ for both spin 
polarization, i.e., the polarizer efficiency is the same for spin "+" and spin "-" neutrons, and the spin-flipper in front of the analyzer does not affect the beam polarization. The formula for the measured reflectivity $\mathcal{R}$ in Eq. (5) can therefore be developed as a function of the sample's non-spin-flip reflectivities $R_{++}$and $R_{--}$in the following way:

$$
\mathcal{R}=\frac{1}{4}\left[\left(1+P_{i}\right) \cdot\left(1+P_{f}\right) \cdot R_{++}+\left(1-P_{i}\right) \cdot\left(1-P_{f}\right) \cdot R_{--}\right] .
$$

In the remanent state $\left(\mu_{0} H=1 \mathrm{mT}\right)$ the sample produces much stronger $R_{--}$reflectivity than $R_{++}$over the whole range of angles of incidence $\alpha_{i}$ [cf. Fig. 6(a)]. In that case, the measured spin-flip reflectivities can be written:

$$
\begin{aligned}
& \mathcal{R}_{+-}=\frac{1}{4}\left(1-\left|P_{i}\right|\right) \cdot\left(1+\left|P_{f}\right|\right) \cdot R_{--}, \\
& \mathcal{R}_{-+}=\frac{1}{4}\left(1+\left|P_{i}\right|\right) \cdot\left(1-\left|P_{f}\right|\right) \cdot R_{--} .
\end{aligned}
$$

In the same way, at saturation $\left(\mu_{0} H=25 \mathrm{mT}\right)$ the sample produces much stronger $R_{++}$reflectivity than $R_{--}$and the measured spin-flip reflectivities are

$$
\begin{aligned}
& \mathcal{R}_{+-}=\frac{1}{4}\left(1+\left|P_{i}\right|\right) \cdot\left(1-\left|P_{f}\right|\right) \cdot R_{++}, \\
& \mathcal{R}_{-+}=\frac{1}{4}\left(1-\left|P_{i}\right|\right) \cdot\left(1+\left|P_{f}\right|\right) \cdot R_{++} .
\end{aligned}
$$

A quick inspection of those formulas shows that, if $\left|P_{i}\right|$ $<\left|P_{f}\right|, \mathcal{R}_{+-}>\mathcal{R}_{-+}$in the remanent state and that $\mathcal{R}_{+-}<\mathcal{R}_{-+}$ at saturation. This is effectively what is observed in Figs. 6(a) and 6(d). At the two other fields, $\mathcal{R}_{+-}>\mathcal{R}_{-+}$in the angular ranges for which $\mathcal{R}_{++} \ll \mathcal{R}_{--}$and $\mathcal{R}_{+-}<\mathcal{R}_{-+}$in the angular ranges for which $\mathcal{R}_{--} \ll \mathcal{R}_{++}$, showing that the relation $\left|P_{i}\right|<\left|P_{f}\right|$ holds at all applied fields. Adjusting the values of $\left|P_{i}\right|$ and $\left|P_{f}\right|$ for the simulated spin-flip reflectivities to meet the data lead to $\left|P_{i}\right|=0.85,0.91,0.91$ and 0.93 at $\mu_{0} H=1.0$, $3.8,5.6$, and $25 \mathrm{mT}$, respectively, and $\left|P_{f}\right|=0.945$ at all applied fields except at $\mu_{0} H=5.6 \mathrm{mT}$ where it is equal to 0.93 . There is no reason for the analyzer efficiency to be smaller at this intermediate field value. This comes from a reduced efficiency of the spin-flipper to a value of 0.98 , due to the fact that the stray fields of the electromagnet enter in conflict with the compensation field in the spin-flipper.

The specular reflectivity data were analyzed according to the formula in Sec. II B. The reflectivity is proportional to the rate of incoming neutrons illuminating the surface of the sample. The illumination effects were determined from ray tracing calculations. They depend on the opening of the two slits before the sample, the size of the sample and its inclination angle. ${ }^{73}$ The neutrons hitting the surface of the sample do not arrive all with the same angle of incidence $\alpha_{i}$ and the same wavelength $\lambda$. These are taken into account assuming a Gaussian-like resolution in $Q_{\perp}$, whose width is determined by the resolution in wavelength, the divergence of the beam and the inclination angle of the sample. The simulations were
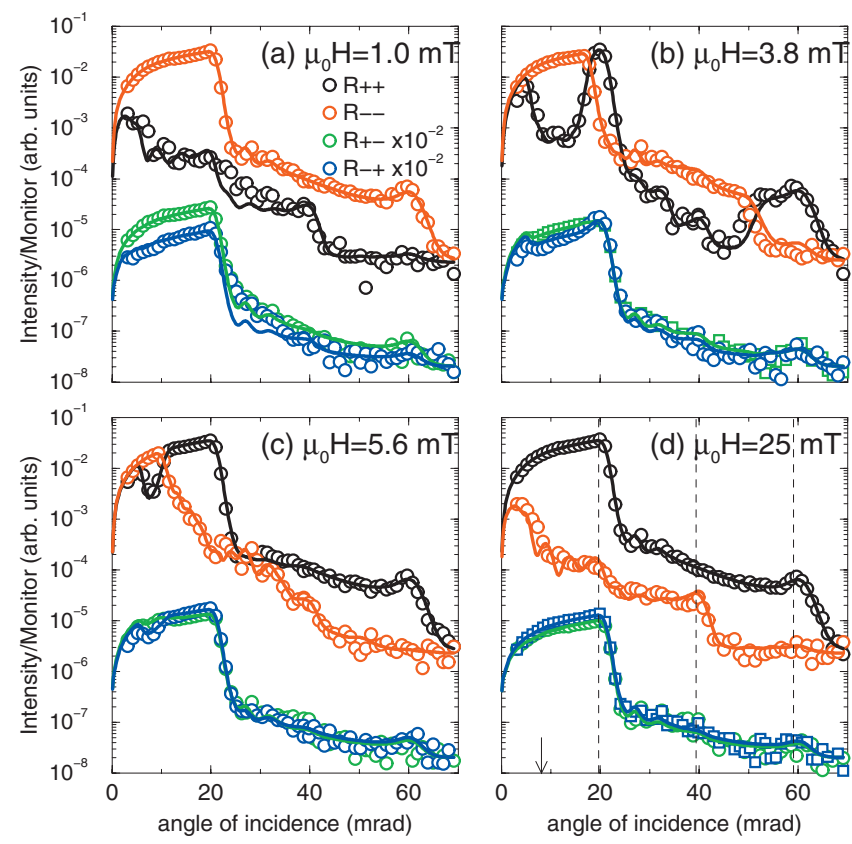

FIG. 6. (Color) Intensities measured along the specular line during remagnetization of a remanent polarizing supermirror after having saturated it in a field of $0.5 \mathrm{~T}$ opposite to the guiding field of the neutron beam. The points are the measurements and the lines are fits to the data according to the formula in Sec. II B. For the purpose of clarity, the spin flip intensities are scaled down by two orders of magnitude. The magnetizations of the bottommost thinnest $\mathrm{FeCoV}$ layers flip at first along the applied field. At $\mu_{0} H=1.0,3.8,5.6$, and $25 \mathrm{mT}$, respectively, 0, 48, 94, and, 100 layer magnetizations have flipped along the applied field. The other fit parameters are given in Sec. V A. In (d), the arrow points at the critical angle of total reflection of "+" neutrons on an $\mathrm{FeCoV}$ surface and the three dashed lines are positioned at one, two, and three times the critical angle of good reflection of "+" neutrons by the supermirror.

convoluted by this resolution function. Also, all simulations were multiplied by a recalibration factor to meet the plateau of total reflection and a constant background was added.

The off-specular data were analyzed according to the formalism presented in Sec. II C. The scattering from magnetic correlations and interfacial roughness was analyzed using the statistical models described in Secs. II D and II E, respectively. The differential scattering cross section in Eq. (21) was then doubly convoluted by Gaussian resolution functions in $\alpha_{i}$ and $\alpha_{f}$ of 2 mrad FWHM.

Three empirical recalibration factors were adjusted to the data, one for the reflectivity, one for the off-specular scattering from structural roughness and another one for the magnetic scattering from lateral magnetic correlations. This comes from the fact that the reflectivity is calculated exactly and that the roughness and magnetic lateral correlation lengths cannot be determined exactly in this experiment (see Sec. V B). Note, however, that the same recalibration factor was used for the two magnetic scattering cross sections in Eq. (22), allowing a precise determination of the parameters $\Phi_{l}$ and $\omega_{l}$ describing the amplitudes of the magnetic fluctuations.

A proper scaling between reflectivity and both types of diffuse scatterings (structural and magnetic) can in principle 
be determined if one calculates the reflectivity within the DWBA and from the knowledge of $S_{\mathrm{coh}}=l_{x} \cdot l_{y}$, projection on the sample surface of the coherence volume of the beam (Sec. II A), entering in the Fourier transforms of the pair correlation functions Eqs. (22) and (25). The specular reflectivity peak has a Gaussian dependence as a function of $Q_{x}$ whose FWHM $\delta Q_{x}$ leads to $l_{x}[\mu \mathrm{m}]=2 \pi / \delta Q_{x}$ $=137 /\left(\alpha_{i}[\mathrm{mrad}]\right)$ along the specular line as a function of the angle of incidence $\alpha_{i}$.

\section{MEASUREMENTS AND ANALYSIS}

\section{A. Measurements along the specular line}

As a first step of our investigation we interpret the data along the specular line extracted from the whole set of data shown in the next subsection. This gives access to the laterally averaged state of the sample around which the fluctuations will be introduced in order to reproduce the offspecular intensities in the next subsection.

In Fig. 6 the four spin channels of polarized neutron reflectivity with polarization analysis are presented at four representative fields along the remagnetization process of the sample after having saturated it in a field of $-0.5 \mathrm{~T}$. The lines are fits to the data according to Eq. (5) taking into account illumination and resolution effects as described in the preceding subsection.

At $\mu_{0} H=1.0 \mathrm{mT}$, the sample is in the remanent state, i.e., all the magnetizations inside the $\mathrm{FeCoV}$ layers are oriented antiparallel to the applied field: The $\mathcal{R}_{--}$intensities are high and the $\mathcal{R}_{++}$intensities are low. At $25 \mathrm{mT}$, the sample is in a saturated state, with all the magnetizations oriented along the applied field, leading to high $\mathcal{R}_{++}$intensities and low $\mathcal{R}_{--}$. Note also in the majority channels (i.e., $\mathcal{R}_{--}$in the remanent state and $\mathcal{R}_{++}$in the saturated one) the sharp drops of the intensities not only at the effective critical angle of the supermirror but also at three times the effective critical angle. No intensity drop is present at two times this angle. This comes from the fact that in a big amount of all the bilayers the $\mathrm{FeCoV}$ thicknesses are equal to the TiN ones (cf. second paragraph of Sec. III) and an extinction rule forbids all even order Bragg peaks if the magnetic potential is spatially uniform inside the $\mathrm{FeCoV}$ layers. This extinction of even order peaks is expected due to the fact that each bilayer contains two interfaces whose reflections are phase shifted by a factor of $\pi$. Such symmetric superlattices are sometimes used in polarized neutron reflectivity when the interface magnetic structure has to be probed (see, e.g., Ref. 18).

The magnetization reversal does not happen via a single flip of all the layers at the same coercive field: at $\mu_{0} H=3.8$ and $5.6 \mathrm{mT}$ angular ranges of high $\mathcal{R}_{++}$and high $\mathcal{R}_{--}$coexist. Inside the plateau of good reflection, as the field is increased, the higher angle part of the (--) channel gets strongly reduced and is replaced by high $(++)$ reflectivity. This shows that the thinnest FeCoV layers have lower coercivities than the thicker ones. ${ }^{34}$ From the fits we deduce that, out of the $100 \mathrm{FeCoV}$ layer magnetizations, 48 of them have flipped along the field at $3.8 \mathrm{mT}$ and 94 at $5.6 \mathrm{mT}$. The number $N$ of flipped $\mathrm{FeCoV}$ magnetizations as a function of the applied field $\mu_{0} \mathbf{H}$ and the deduced coercivity $H_{C}$ as a func-
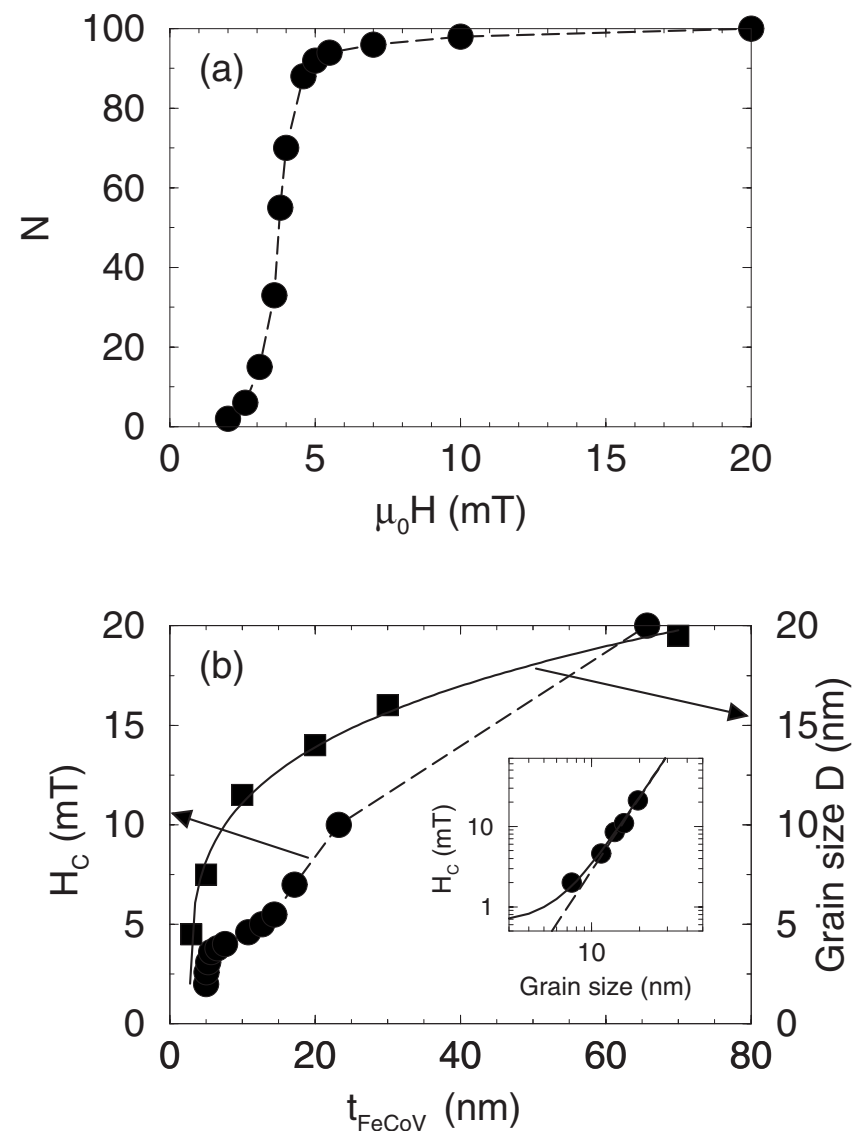

FIG. 7. (a) As the field is reversed after magnetic saturation of the sample, the magnetization reversal of the FeCoV layers proceeds sequentially from the bottom to the top. The number $N$ of reversed $\mathrm{FeCoV}$ layer magnetizations is displayed as a function of the applied field. (b) Circles: Coercive field $H_{C}$ of $\mathrm{FeCoV}$ layer magnetizations deduced from (a) as a function of the layer thickness. Squares: Grain size $D$ as a function of layer thickness as deduced from x-ray diffraction in Ref. 39. Solid line: Fit to the data leading to the relation $D \propto\left(t_{\mathrm{FeCoV}}-2.8\right)^{0.26 \pm 0.2}$. Inset: Points: Coercive field as a function of grain size deduced from the two other curves. Solid line: Fit to the data leading to $H_{C}=0.6+7.1 \times D^{3}$. Dashed line: Simulation $H_{C}=7.1 \times D^{3}$.

tion of FeCoV layer thickness $t_{\mathrm{FeCoV}}$ are depicted in Fig. 7.

Careful analysis of the data led to further structural and magnetic information. The measured spin flip intensities are reproduced by only taking into account the polarizing and flipping inefficiencies given in Sec. IV C. No spin-flip reflectivity is induced by the sample. We therefore conclude that all the net magnetizations lay either parallel or antiparallel to the applied field. The remagnetization process does not happen via in-plane rotation of the magnetizations.

The intensities beyond the plateau of total reflection show a gradual decay as a function of the angle of incidence, that we interpret by the presence of interfacial roughness. This decay is, however, faster at smaller angles than at higher, with even an inversion of the slope at angles between 55 and $60 \mathrm{mrad}$ (see $\mathcal{R}_{--}$at $\mu_{0} H=1.0 \mathrm{mT}$, and $\mathcal{R}_{++}$at the three other fields of Fig. 6). We reproduce this effect assuming smaller roughness amplitude of the bottommost interfaces 
$(0.34 \mathrm{~nm} \mathrm{rms})$ than the topmost $(1.26 \mathrm{~nm} \mathrm{rms})$, with a power law variation as a function of the number of interfaces (counted from the substrate's interface) with an exponent equal to 0.278 . A linear variation does not reproduce very well the upturn of the intensities at the biggest angles. A power law with exponent smaller than one provides a faster increase in roughness amplitudes for the bottommost and a slower one for the topmost layers than a linear law. Similar behavior, i.e., a faster increase of roughness amplitudes at the bottommost interfaces than at the topmost, was reported in FeCoV/TiZr supermirrors. ${ }^{74}$

The relatively high intensities around $4 \mathrm{mrad}$ in the minority channels (i.e., $\mathcal{R}_{++}$channel in the remanent state and $\mathcal{R}_{--}$channel in the saturated state) are due to the substrate that introduces a potential mismatch with the TiN layer. The Gd layer between the supermirror structure and the substrate reduces the importance of this peak and introduces the oscillations seen in those channels.

At the exception of the minority channels in the 20 to $40 \mathrm{mrad}$ range (see next paragraph), all simulated intensities overestimate the measured data. A good matching is obtained by reducing by $13 \%$ all atomic densities with respect to their nominal values. In Ref. 39, x-ray diffraction shows that the intake of nitrogen atoms shifts the [110] texture peak of $\mathrm{FeCoV}$ to lower angles (from $45^{\circ}$ to $42.5^{\circ}$ ), indicating a larger lattice parameter (by 6\%) and lower atomic density (by $16 \%$ ), in quantitative agreement with our result.

Assuming perfect matching between the "-" neutron interaction potential with $\mathrm{FeCoV}$ and the neutron interaction potential with TiN leads systematically to a strong underestimation of the intensities in the minority channels between 20 and $40 \mathrm{mrad}$. Moreover, the presence of a sharp intensity drop in those channels at $40 \mathrm{mrad}$ is, even qualitatively, not reproduced by the simulations. Layer-uniform modifications or even layer-to-layer variations of the nuclear or magnetic scattering length densities do not improve the fit. Note that the angular range under consideration corresponds to the one for second order Bragg peaks of the supermirror structure. As pointed out in the third paragraph of this subsection, evenorder Bragg peaks are forbidden in a symmetric multilayer if the magnetic potential is spatially uniform inside the $\mathrm{FeCoV}$ layers. Breaking this assumption by replacing in the simulations the top and bottom parts of the $\mathrm{FeCoV}$ layers by layers of same nuclear scattering length density than $\mathrm{FeCoV}$ but zero magnetic scattering length density (i.e., "magnetically dead" layers) results in a drastic improvement of the fit. A perfect agreement between data and simulation is obtained by assuming different thicknesses of the top and bottom dead layers, i.e., $0.15 \mathrm{~nm}$ and $0.4 \mathrm{~nm}$. The fit does not allow us to distinguish which of the top and bottom dead layers are thicker than the others. Those values are in good agreement with the value of $0.21 \mathrm{~nm}$ found by Senthil-Kumar et al. ${ }^{39}$ in the same system from saturation magnetization measurements.

At this stage of the fit, in the remanent state, the $\mathcal{R}_{++}$ intensity below the effective critical angle of the supermirror $(\approx 20 \mathrm{mrad})$ and above $10 \mathrm{mrad}$ is still underestimated by the simulations. The three other channels and all the channels at the other fields are well reproduced. An increase of the simulated $\mathcal{R}_{++}$at remanence that does not affect the simula-

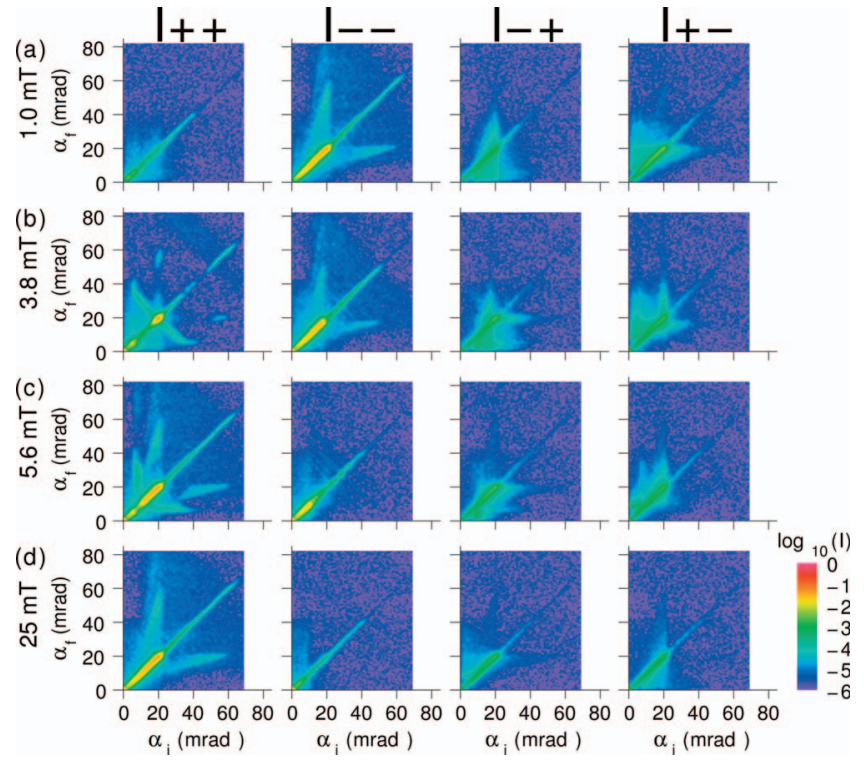

FIG. 8. (Color) Spin resolved reflectivity and off-specular scattering measured after saturation of the sample in a negative field at four applied fields (a) $\mu_{0} H=1 \mathrm{mT}$, (b) $3.8 \mathrm{mT}$, (c) $5.6 \mathrm{mT}$, and (d) $25 \mathrm{mT}$.

tion at the other fields can be obtained by increasing the contrast between $\mathrm{FeCoV}$ and TiN only when FeCoV layers are involved with magnetizations that have not yet reversed along the field. This is obtained by assuming a $10 \%$ reduction of the net magnetization in the $\mathrm{FeCoV}$ layers that have not yet flipped. This is a first hint that, on a length scale smaller than the lateral coherence length of the beam, some magnetic fluctuations exist in the layers that have not yet flipped along the applied field.

The lines in Fig. 6 represent the simulations at this final stage of the fit.

\section{B. Off-specular scattering}

The full set of data, i.e., specular reflectivity plus offspecular scattering, measured at the four fields considered above, is shown in Fig. 8. Along the main diagonals, one recognizes the specular intensities depicted in Fig. 6.

As a help to understand the measured data, we also calculate the probability densities $W_{+}$and $W_{-}$to find spin up and spin down neutrons in the multilayer stack as a function of the angle of incidence $\alpha_{i}$. They are obtained by projecting the ket, solution of the Schrödinger equation for the average potential, on the bras representing spin up and spin down neutrons respectively. Using the propagator in layer $l$ introduced in Eq. (2), they can be calculated using the following formulas:

$$
\begin{gathered}
W_{+}\left(z, \alpha_{i}\right)=\sum_{l}\left|\left\langle+\left|\hat{S}_{i l}(z)\right|+\right\rangle\right|^{2}, \\
W_{-}\left(z, \alpha_{i}\right)=\sum_{l}\left|\left\langle-\left|\hat{S}_{i l}(z)\right|-\right\rangle\right|^{2}
\end{gathered}
$$

and are represented in Fig. 9 for the 4 laterally averaged states determined in the previous subsection. 


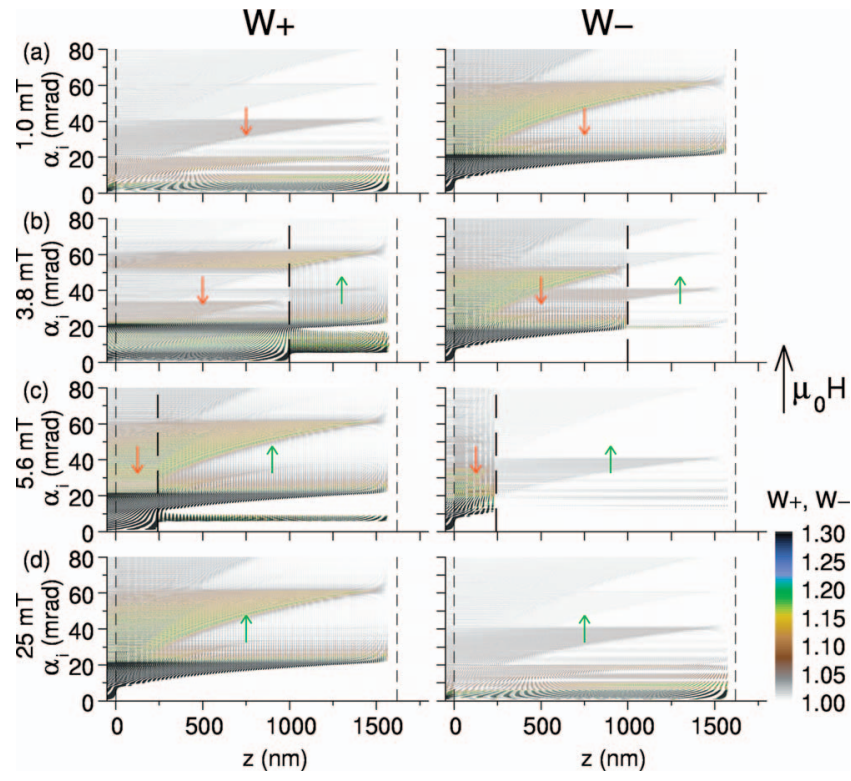

FIG. 9. (Color) Probability densities to find "+" $\left(W_{+}\right)$and "-_" $\left(W_{-}\right)$neutrons in the supermirror calculated as a function of depth $z$ in the multilayer and angle of incidence $\alpha_{i}$. The calculations are performed for the four average structural and magnetic configurations deduced from the specular reflectivity analysis at the four fields of Fig. 8. The two densities are plotted on the same arbitrary unit. The three dashed vertical lines in each picture represent the interface with air $(z=0)$, the interface between regions of nonflipped and flipped layer magnetizations, and the interface with the substrate. The red (green) arrows denote the regions inside which the magnetizations lay opposite (along) the applied magnetic field $\mu_{0} \mathbf{H}$.

In Fig. 10, the simulation of the data is presented with the parameters given below. It is the sum of the simulations of specular reflectivity, of off-specular scattering from interfacial roughness correlations, from lateral correlations of the magnetic fluctuations perpendicular to the applied field, and from lateral correlations of the magnetic fluctuations parallel to $\mu_{0} \mathbf{H}$ (cf. Sec. II). The three simulations of off-specular scattering within the DWBA are presented separately in Figs. 11 to 13 , respectively. Illumination and resolution effects are also taken into account as described in Sec. IV C.

\section{Scattering from interfacial roughness correlations}

In the remanent state, strong off-specular scattering is seen in the $I_{--}$channel for which a strong contrast between the layers exist. As the field is increased and the bottommost layers begin to flip along the applied field, the disappearance (appearance) of specular intensity in the $I_{--}\left(I_{++}\right)$channel is accompanied by the disappearance (appearance) of the scattering intensity in the same channel. Thus, the intensities of scattering depend, like the specular reflectivities, on the contrast between neighboring $\mathrm{FeCoV}$ and $\mathrm{Ti}: \mathrm{N}$ layers. They are therefore due to structural roughness at the interfaces. The scattering from interfaces with $\mathrm{FeCoV}$ layers with magnetizations along the field is mainly seen in the $I_{++}$channel, and the scattering from those with magnetizations antiparallel the field is seen in the $I_{--}$channel.
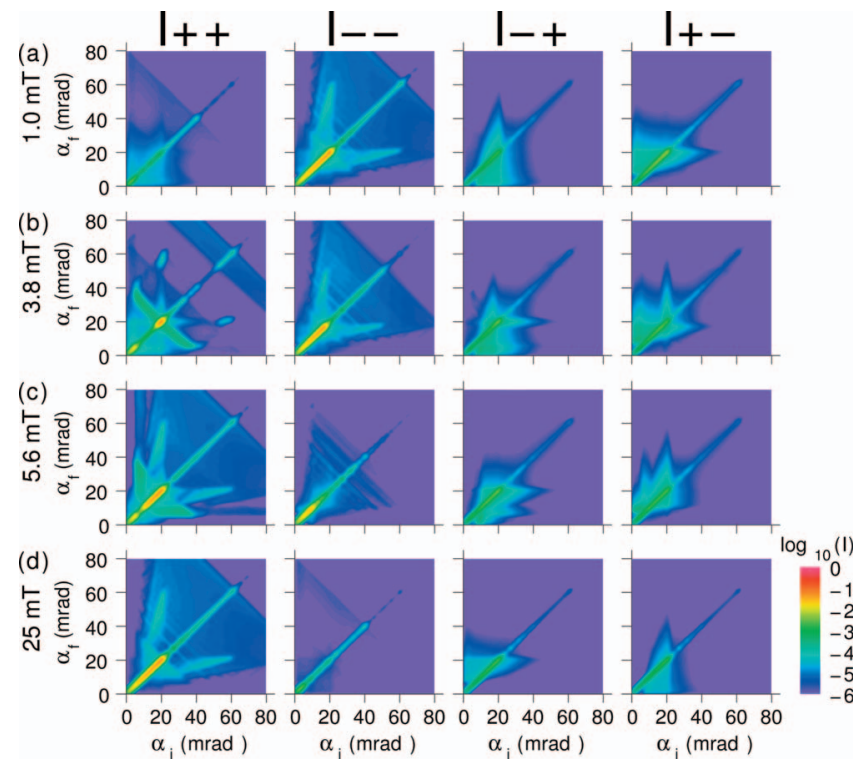

FIG. 10. (Color) Simulation of the reflectivity and off-specular scattering data of Fig. 8 in the framework of the distorted wave Born approximation (DWBA). The simulation of scattering results from the sum of the scattering from interfacial roughness (Fig. 11), of the one from fluctuations of the magnetizations perpendicular to the applied field (Fig. 12), and from parallel magnetic fluctuations (Fig. 13).

At $3.8 \mathrm{mT}$ in the $I_{++}$channel, the scattering from roughness is concentrated around a line perpendicular to the specular line and crossing it at around $20 \mathrm{mrad}$ where the specular

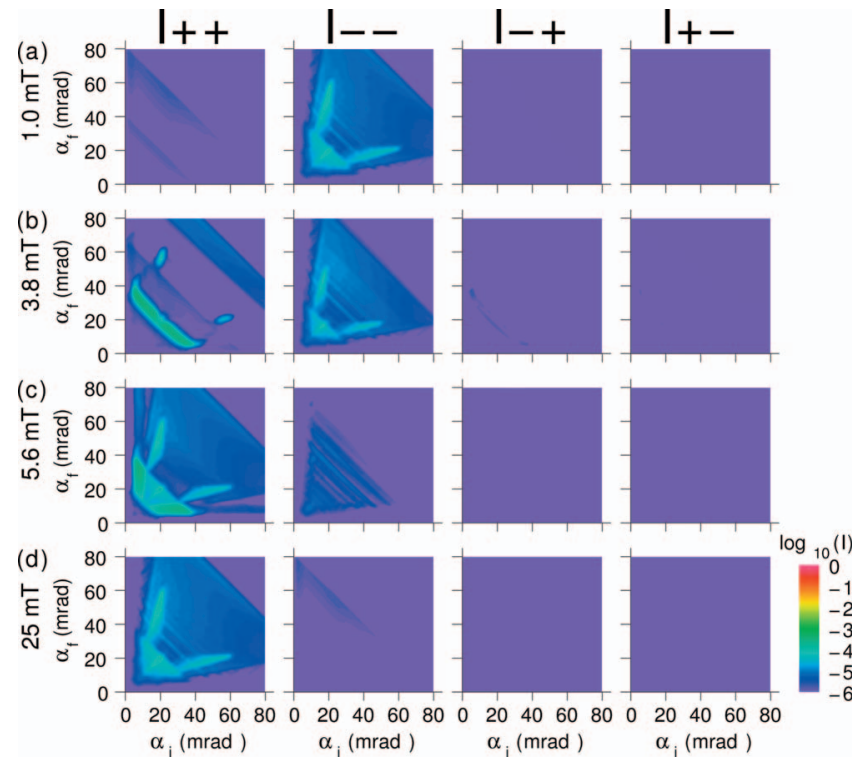

FIG. 11. (Color) Part of the DWBA simulation of off-specular scattering originating from interfacial roughness and calculated using the structure factor in Eq. (25). For this simulation we took the rms roughness amplitudes as deduced from the fit of the specular reflectivity, the same lateral correlation length $\xi_{l}^{R}$ for all interfaces $l$ $\left(\xi_{l}^{R} \ll 1 \mu \mathrm{m}\right)$ and $\xi_{\perp}^{R}$ very large with respect to the thickness of the entire multilayer stack. Note that scattering from interfacial roughness does not produce any spin-flip signal. The latter is solely due to transverse magnetic fluctuations. 
intensity is at its maximum. This so-called "Bragg sheet" shows that the in-plane roughness profile is vertically correlated, i.e., that it replicates itself from interface to interface $\left[\xi_{\perp}^{M}\right.$ in $C_{l l^{\prime}}^{R}$ of Eq. (25) large with respect to the entire multilayer thickness]. Note also the "bending" of the Bragg sheet at $\alpha_{i}$ and $\alpha_{f}$ around $8 \mathrm{mrad}$, equal to the critical angle of total reflection for "+" neutrons of $\mathrm{FeCoV}$. This can be explained by considering the probability density to find a "+ neutron" in the layers whose magnetizations lie along the applied field [region labeled by a green arrow in Fig. 9(b)]: Below this angle, no "+ neutron" can be observed. This is due to the fact that, below this critical angle, the "+ neutron" wave is evanescent. This effect is even more dramatic for the Bragg sheet in $I_{++}$at $5.6 \mathrm{mT}$. Here the Bragg sheet is completely bent and lies parallel to the $\alpha_{i}$ or $\alpha_{f}$ axis depending on $\alpha_{i}$ larger or smaller than $\alpha_{f}$, respectively.

Almost all of the rest of the non-spin-flip off-specular data also arises from scattering by vertically correlated roughness, as confirmed in the simulations of Figs. 10 and 11. Although those intensities do not appear at $\alpha_{i}$ and $\alpha_{f}$ values close to total external reflection, here also they do not have the usual Bragg-sheet form, i.e., they are not distributed in angular ranges parallel to a line of constant $\alpha_{i}+\alpha_{f}$ value. This can be explained by considering again the probability densities. For example, let us take the $I_{--}$scattering in the remanent state. At remanence $W_{-}$is prominent in the $\alpha=8$-to- $22 \mathrm{mrad}$ angular range. The sample is probed deeper by "-" neutrons as the angle of incidence is increased. At a fixed angle of incidence $\alpha_{i}$, the interface between the neutron probed and nonprobed regions is given by the $\mathrm{FeCoV} / \mathrm{TI}$ : N bilayer thickness $t$ fulfilling the Bragg condition for reflection $t=n \cdot \lambda / 2 \alpha$ to first order $(n=1)$. Another range of prominent illumination lies from 8 to $60 \mathrm{mrad}$ with the same interface at three times the former angle and fulfilling the Bragg condition to third order $(n=3)$. As pointed out in the former subsection, the Bragg condition to even order is not illuminated. Off-specular scattering should therefore be observable at $\alpha_{i}$ and $\alpha_{f}$ lying in one of those ranges. As the "-" neutrons illuminate more and more interfaces as the angle of incidence is increased, the related off-specular scattering intensity should also increase with $\alpha_{i}$ and $\alpha_{f}$. This is what is observed in Fig. 8(a) and simulated in Figs. 10(a) and 11(a). The same argument holds for the explanation for the scattering in the $I_{--}$channel at $3.8 \mathrm{mT}$ [Fig. 8(b)] and in the rest of the scattering in the $I_{++}$channels at 3.8 and 5.6 [Fig. 8(c)] mT.

From the above discussion one can conclude that the shape of the off-specular scattering from interfacial roughness and its intensity distribution are solely determined by dynamical effects. The intensity distributions are well reproduced by the sequence of roughness amplitudes deduced in the preceding subsection from the specular reflectivity but are completely independent of the chosen value for the lateral correlation $\xi_{l}^{R}$ (taken equal for all interfaces $l$ ) in Eq. (25), provided that it is much smaller than, say, one $\mu \mathrm{m}$. It is therefore not possible to determine the lateral correlation length in the present experimental geometry.

Note also that the data are very well reproduced by the simulations within the small $Q_{\perp} \sigma$ approximation of the DWBA. As a matter of fact, the bottom- (top-) most inter- faces contributing to the scattering at the biggest (smallest) $Q_{\perp}$ values are the ones of smallest (biggest) roughness amplitudes so that the relation $Q_{\perp} \cdot \sigma<1$ is always fulfilled.

\section{Scattering from lateral magnetic correlations}

A strong spin-flip off-specular scattering is also observed (cf. $I_{-+}$and $I_{+-}$channels in Fig. 8). It originates from fluctuations of the component of the magnetization perpendicular to the quantization axis of the neutrons, i.e., perpendicular to the applied magnetic field $\mu_{0} \mathbf{H}$.

Note that, although spin-flip intensity is observed offspecular, such intensity is not observed along the specular direction (apart from the ones due to the inefficiencies of the spin polarizing and analyzing devices, see Sec. IV C). As stated in Sec. II A, specular reflectivity originates from the depth variation of the laterally averaged neutron-matter interaction and off-specular scattering originates from correlations of the lateral fluctuations around this mean value. In our case, the laterally averaged magnetic potential consists of the interaction with layer magnetizations being either parallel or antiparallel to the guide field. Therefore, specular reflectivity is seen in the non-spin-flip channels only.

The off-specular scattering does not have the tendency to arrange along Bragg sheets. It is therefore due to vertically uncorrelated magnetic fluctuations.

In the $I_{-+}\left(I_{+-}\right)$channel, neutrons are incident on the sample surface under the angle $\alpha_{i}$ and spin "-" ("+") and are detected under the angle $\alpha_{f}$ with spin "+" ("-"). The $\alpha_{i}$ and $\alpha_{f}$ values at which the spin-flip off-specular scattering is prominent correspond to $\alpha_{i}$ values at which the reflectivities for "-" and "+" neutrons, respectively, are prominent. This explains the big asymmetry with respect to the specular line of the spin-flip signals. For example, at remanence the $I_{-+}$ $\left(I_{+-}\right)$signal stands mainly below (above) the specular line because the "-" reflectivity is much more prominent than the "+" reflectivity. This asymmetry is inverted at $25 \mathrm{mT}$, the field at which all the magnetizations have flipped along the field inducing strong "+" and low "-" reflectivities.

At the three smallest fields, the $\alpha_{i}$ and $\alpha_{f}$ values at which the spin-flip off-specular scattering is prominent, correspond in Fig. 9 almost exclusively to angles where the probabilities $W_{-}$and $W_{+}$respectively are prominent in the layers whose magnetizations point antiparallel the applied field. Almost all the spin-flip off-specular scattering could be reproduced by the DWBA simulations introducing fluctuations only in the layers that have not yet flipped along the field.

The presence of spin-flip off-specular scattering at $25 \mathrm{mT}$ shows that magnetic fluctuations are also present when the magnetizations have flipped along the applied magnetic field. Magnetic fluctuations are also present in the flipped magnetizations at the three smallest fields. To show this, let us concentrate, for example, on the $I_{-+}$channel. This cannot be shown from the intensity variation along the $\alpha_{i}$ axis, as $W_{-}$in the layers along the field is very weak. $W_{+}$in the layers with magnetizations along the field is maximized in narrow angular ranges around 9 and $20 \mathrm{mrad}$ at $\mu_{0} H=3.8$ and $5.6 \mathrm{mT}$. The weak spin flip scattering at $\alpha_{f}$ lying in those two angular ranges at those two fields can therefore be reproduced only 

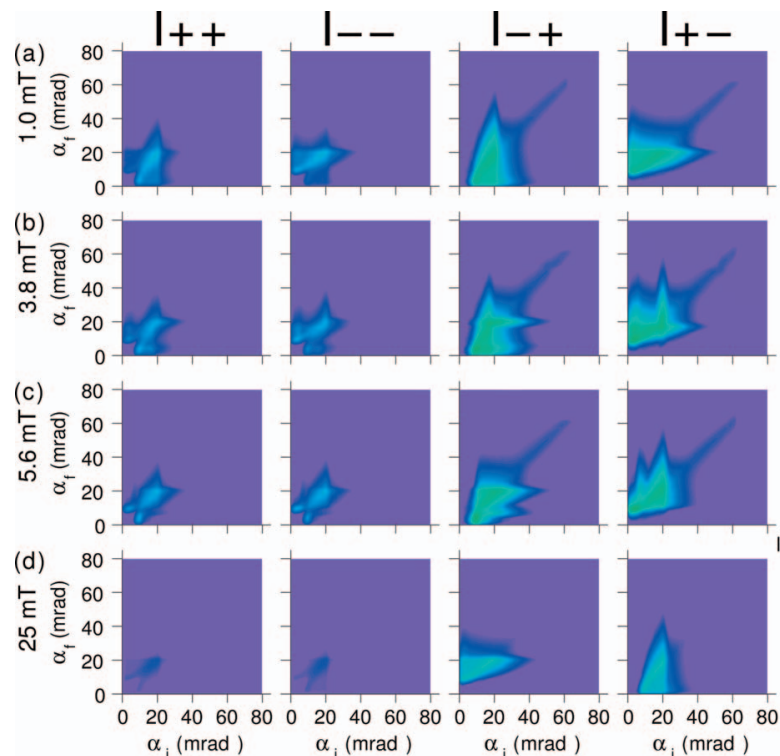

$\alpha_{i}(\operatorname{mrad})$

$\alpha_{i}(\mathrm{mrad})$
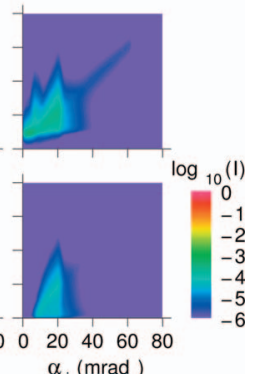

FIG. 12. (Color) Part of the DWBA simulation of off-specular scattering originating from the fluctuations of the layer magnetizations perpendicular to the applied field $\mu_{0} \mathbf{H}$. Those fluctuations produce off-specular scattering in the spin-flip channels. This calculation was performed using the first structure factor in Eq. (22) with the following parameters: $\xi_{l}^{M}=0.2 \mu \mathrm{m}$ the same for all layers $l$ and all fields, $\Phi_{l}=155^{\circ}$ for the nonreversed layer magnetizations and $0^{\circ}$ for the flipped ones for all fields, $\omega_{l}=35^{\circ}$ for all layers at remanence, $\omega_{l}=40^{\circ}$ at $\mu_{0} H=3.8$ and $5.6 \mathrm{mT}$, and $\omega_{l}=15^{\circ}$ at $25 \mathrm{mT}$. Note that the nonzero intensities in the non-spin-flip channels arise from taking into account in the density matrices in Eq. (21) the nonperfect efficiencies of the polarizer and analyzer devices.

assuming that magnetic fluctuations are present also in the layers that have flipped along the field.

A good reproduction of the spin-flip scattering at the three smallest fields (Figs. 10 and 12) was obtained in the following way. To take into account the absence of cross correlations between different layers [i.e., $S_{l l^{\prime}}^{M}=0$ when $l \neq l^{\prime}$ in the first Fourier transform of pair correlation function in Eq. (22)] we took $\xi_{\perp}^{M}=0$. The extension of the scattering along the $Q_{x}$ direction was well reproduced by taking the same lateral correlation length $\xi_{l}^{M}=(0.2 \pm 0.05) \mu \mathrm{m}$ in all layers $l$. In order to remain consistent with the specular reflectivity analysis (Sec. V A) in which a $10 \%$ reduction of the net magnetization with respect to the saturation magnetization is found in the layers with magnetizations that have not yet flipped along the field, we take $\Phi_{l}=155^{\circ}$ in the autocorrelation functions $S_{l l}^{M}$. For the magnetizations that have already flipped we take $\Phi_{l}=0$. Then the overall recalibration factor for magnetic scattering (discussed at the end of Sec. IV C) was adjusted in order to reproduce the measured intensities.

In this way, it was not possible to reproduce the spin-flip intensity at $25 \mathrm{mT}$, as it was found from the specular reflectivity analysis that the average net magnetization in the layers with magnetizations along the applied field are equal to the saturation magnetization. Moreover, it was not possible to reproduce fully the non-spin-flip off-specular scattering at $\alpha_{i}$ and $\alpha_{f}$ angles below $20 \mathrm{mrad}$ assuming only off-specular scattering from roughness. Those missing scattering intensi-
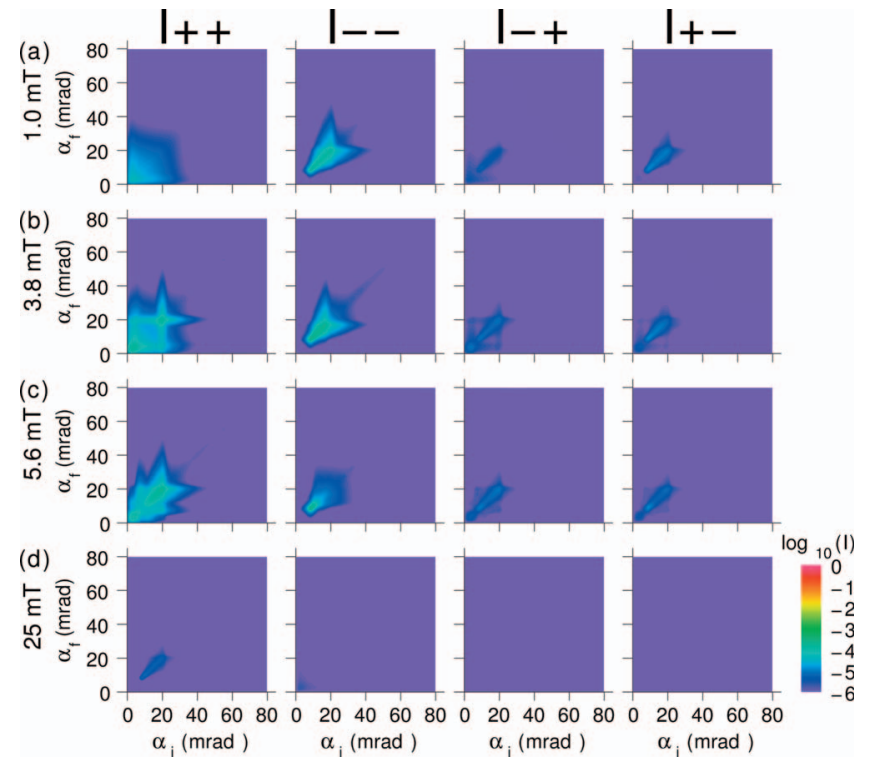

FIG. 13. (Color) Part of the DWBA simulation of off-specular scattering originating from the fluctuations of the layer magnetizations parallel to the applied field $\mu_{0} \mathbf{H}$. Those fluctuations produce off-specular scattering in the non-spin-flip channels. The same parameters as in Fig. 12 were used. Again the nonvanishing intensities in the spin-flip channel arise from taking into account the nonperfect efficiencies of the polarizing and analyzing devices.

ties were obtained assuming fluctuations of the component of the magnetizations parallel to the applied field. In the model of lateral magnetic correlations depicted in Sec. II D, this can be obtained by putting some lateral randomness in the orientation of the magnetization from spin-correlated region to spin-correlated region $\left[\omega_{l} \neq 0\right.$ in $C_{l l}$ and $S_{l l}$ of Eq. (22)]. The introduction of this randomness leads to a nonvanishing structure factor for longitudinal magnetic fluctuation. The intensities were well reproduced by assuming the rms amplitudes $\omega_{l}$ independent of the layer $l$ and equal to $(35 \pm 5)^{\circ}$ at remanence, $(40 \pm 5)^{\circ}$ at $\mu_{0} H=3.8$ and $5.6 \mathrm{mT}$, and $(15 \pm 5)^{\circ}$ at $25 \mathrm{mT}$ (Figs. 10 and 13).

\section{DISCUSSION}

We start here by summarizing the structural and magnetic model of the sample and its magnetic behavior during the magnetization reversal process as obtained from the analysis of the reflectivity and off-specular data. The interfaces show increasing rms roughness amplitudes from the bottommost to the topmost interface, ranging from 0.34 to $1.26 \mathrm{~nm}$. Strong cross-correlations of the roughness profiles are deduced. Due to the limited $Q_{x}$ range accessible with the present instrumental geometry, we are not able to access to their lateral correlation length.

The top and the bottom parts of each of the FeCoV layers show vanishing magnetization. Those "dead layers" are 0.15 and $0.4 \mathrm{~nm}$ thick. It is not possible to distinguish which of the bottom and top "dead layers" is the thickest.

As the external magnetic field $\mu_{0} H$ is reversed after saturating the sample, the magnetization reversal proceeds se- 


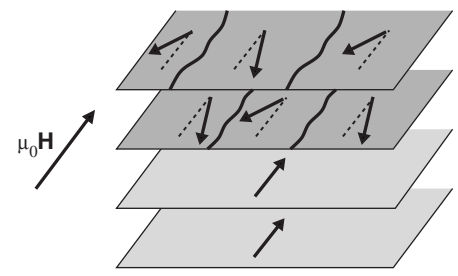

FIG. 14. Sketch of the magnetic microstructure of the supermirror at the magnetization reversal. In the topmost layers, the magnetizations have not yet reversed along the field and $\Phi_{l} \neq 0$, while in the bottommost layers $\Phi_{l}=0$. Here, for the sake of clarity, the random part of the spin misalignment is not sketched.

quentially from the bottommost and thinnest to the topmost and thickest FeCoV layer. In Fig. 7(a) the number of reversed layer magnetization is depicted as a function of $\mu_{0} \mathrm{H}$. At all the fields of measurement, the magnetizations are found either parallel or antiparallel to $\mu_{0} \mathbf{H}$. No component of the net magnetizations perpendicular to $\mu_{0} \mathbf{H}$ was detected. The net magnetizations as deduced from the specular reflectivity measurements are saturated when they are aligned along the field and are reduced by $10 \%$ with respect to their saturation value when they are aligned antiparallel the field. This is the first hint that, at least in the layers that have not reversed their magnetization, lateral fluctuations of the magnetizations exist, i.e., that $\Phi_{l} \neq 0$.

Lateral correlations of the spin misalignment are present inside the magnetic layers, with an average correlation length $\xi_{l}^{M}$ equal to $0.2 \pm 0.05 \mu \mathrm{m}$. The off-specular scattering with polarization analysis allowed us to separate the fluctuations into transverse and longitudinal ones, i.e., into fluctuations of the component of the magnetization perpendicular and parallel to the applied field direction, respectively. Those two types of fluctuations are interpreted in the following way: From one laterally correlated region to the other, two types of spin misalignment coexist in the nonreversed layers, i.e., a nonrandom one (given by the parameter $\Phi_{l}=155^{\circ}$ ) onto which a Gaussian random fluctuation of the misalignment is superimposed with rms width $\omega_{l}$. In the reversed layers only the random fluctuations are present. Both types of misalignments are not cross-correlated, i.e., they do not correlate from ferromagnetic layer to ferromagnetic layer, as can be expected in a layered system without magnetic coupling. A sketch of the magnetic microstructure of the sample is given in Fig. 14.

\section{A. Coercivity}

The magnetization reversal model as described above, i.e., an increase of the coercive field as a function of the FeCoV layer thickness, can be understood from the nanocrystalline nature of the layers. Senthil-Kumar et al. ${ }^{39}$ deduced the grain size in FeCoV layers from the (200) peaks of bcc $\mathrm{FeCoV}$ in $\mathrm{FeCoV} / \mathrm{TiN}_{x}$ multilayers of equal $\mathrm{FeCoV} / \mathrm{TiN}_{x}$ bilayer thicknesses. The dependence of the grain size $D$ on FeCoV thickness $t_{\mathrm{FeCoV}}$ has been deduced [Fig. 4(b) of Ref. 39] and is reported in Fig. 7(b). In the same plot, we represent the variation of the coercive field $H_{C}$ as a function of $t_{\mathrm{FeCoV}}$ deduced from Fig. 7(a). From those two plots, it is possible to deduce the variation of $H_{C}$ as a function of grain size [inset of Fig. 7(b)]. The coercive field $H_{C}$ increases steeply with grain size $D$.

A steep increase of the coercive field as a function of grain size is a general feature of soft magnetic nanocrystalline alloys ${ }^{35,75,76}$ and can be explained in the framework of the random anisotropy model originally developed for amorphous ferromagnets. ${ }^{77}$ The low coercive field in nanocrystalline alloys is the result of the competition between uniaxial anisotropy of strength $K_{1}$ varying randomly from grain to grain and exchange coupling $A$ that tends to align the magnetization of neighboring grains along a common direction. When the grains are big enough, domain walls of thickness of the order of $l_{W}=\sqrt{A / K_{1}}$ can form at the interface between grains and the magnetizations tend to follow the random anisotropy. Below a grain size $D$ of the order of $l_{W}, D$ is so small that the idea of a magnetization transition layer at the grain boundaries loses significance. When $D \ll l_{W}$, exchange coupling leads to the formation of regions of size $D_{\text {eff }} \gg D$ inside which the magnetization is approximately uniform (see bottom part of Fig. 5). Each region is characterized by an anisotropy $K_{\text {eff }} \ll K_{1}$, varying at random from region to region. Consequently one has $D_{\text {eff }} \propto \sqrt{A / K_{\text {eff }}}$ because this is the minimum length over which one can have magnetization nonuniformities for the given anisotropy $K_{\text {eff. }} K_{\text {eff }}$ represents the effective anisotropy of a region uniformly magnetized and containing a large number $N$ of grains. Because the grain orientation is random, on the average we expect no anisotropy at all. However, the actual anisotropy, being the average of $N$ random contributions of the order of $K_{1}$, will deviate from zero by an amount of $K_{1} / \sqrt{N}$ in a random direction. In our case $N$ is of the order of $\left(D_{\text {eff }} / D\right)^{3}$, and consequently $K_{\text {eff }} \propto K_{1}\left(D / D_{\text {eff }}\right)^{3 / 2}$. From the two above proportionality relations involving $K_{\text {eff }}$ we obtain $K_{\text {eff }} \propto K_{1}\left(D / l_{W}\right)^{6}$. The effective anisotropy decreases abruptly with grain size.

In our system, a uniform uniaxial anisotropy $K_{u}$, induced by the magnetoelastic interactions described in Sec. III, superimposes to the random anisotropy so that the effective anisotropy is now well described by $K_{\text {eff }}$ $=\sqrt{K_{u}^{2}+K_{1}^{2}\left(D / D_{\text {eff }}\right)^{3}}$. Here again, $D_{\text {eff }}$ is self-consistently related to the effective anisotropy by $D_{\text {eff }} \propto \sqrt{A / K_{\text {eff }}}$. In the general case, the effective anisotropy has to be determined by numerical iterations. In the limiting case of dominating uniform anisotropy, the effective anisotropy is given by $K_{\text {eff }}$ $\propto K_{u}+\beta D^{3}$ where $\beta$ is a constant. ${ }^{75}$ Neglecting the magnetostatic interactions, the coercive field $H_{C}$ should also follow such a law.

Fitting the variation of the coercive field as a function of the grain size to a law deduced from the random anisotropy model with uniform uniaxial anisotropy reproduces perfectly the data [see solid line in the insert of Fig. 7(b)]. Note that the coercive field deviates from a pure $H_{C} \propto D^{3}$ relation for a grain size $D$ below $10 \mathrm{~nm}$.

\section{B. Spin misalignment}

Two types of spin misalignment are deduced, random and not random, both types being present in the layers whose 
magnetization have not yet reversed along the applied field, only the random one in the layers with reversed magnetizations. We attribute the average spin misalignment to the applied field that acts against the uniform anisotropy creating an energy barrier for the spins to flip along $\mu_{0} \mathbf{H}$. Due to exchange interactions, the system reacts by creating neighboring regions of constant magnetizations that make sequentially angles $\Phi_{l}$ and $-\Phi_{l}$ with respect to $\mu_{0} \mathbf{H}$. On top of that, the nanostructure of the layers with random orientation of the grains introduces some randomness in $\varphi_{l}$ characterized by the rms amplitude $\omega_{l}$.

The effective length $D_{\text {eff }}$ of the random anisotropy model, size of the regions inside which the magnetization stays approximately uniform, can very well be associated to the lateral correlation length of the spin misalignment $\xi_{l}^{M}$ introduced in Sec. II D and deduced in Sec. V B 2. $D_{\text {eff }}$ can also be deduced from the random anisotropy model with dominant uniform uniaxial anisotropy, leading to the relation $D_{\text {eff }} \propto D^{-3 / 2}$ for big enough grain size. However, within the uncertainty of its estimation, we did not deduce any variation of $\xi_{l}^{M}$ with the layer $l$. Two complementary explanations can be given. First, the present scattering geometry does not allow us to determine precisely lateral correlations lengths much smaller than $0.5 \mu \mathrm{m}$. Second, $70 \%$ of the FeCoV layers, representing $60 \%$ of the volume of the multilayer stack, have thicknesses smaller than $10 \mathrm{~nm}$ in which the grains have sizes below $12 \mathrm{~nm}$ [Fig. 7(b)]. For those small grain sizes, the coercivity, being dominated by the uniform uniaxial anisotropy, deviates from the $D^{3}$ law [cf. inset of Fig. 7(b)] and the effective correlation length does not vary too much. Those two arguments explain why the data were well fitted using an average lateral correlation length of the spin misalignment equal to $\xi_{l}^{M}=0.2 \pm 0.05 \mu \mathrm{m}$ inside all layers $l$.

\section{CONCLUSIONS AND OUTLOOK}

We have investigated the structural and magnetic correlations during the remagnetization process of a gradient nanocrystalline multilayer with uniform uniaxial anisotropy by reflectometry and off-specular scattering of neutrons with polarization analysis. The off-specular data were analyzed within the distorted wave Born approximation (DWBA) including interfacial roughness and lateral correlations of the spin misalignment. This method of measurement and data analysis has allowed to dig out unique informations, not attainable by other techniques.

The magnetization reversal proceeds sequentially from the bottommost and thinnest ferromagnetic layer to the topmost and thickest one. It is quantitatively explained by the increase of the grain size with layer thickness within the random anisotropy model including dominant macroscopic uniaxial anisotropy. The off-specular measurements with polarization analysis allowed us to deduce two types of lateral spin misalignment, random and not random, the first one due to the random orientation of the grains, the second one in the nonreversed layers only and due to the applied magnetic field acting against the uniaxial anisotropy.

For both magnetic and roughness fluctuations, the correlation lengths could not be precisely determined, due to the present instrumental geometry. Two methods can be used to have a precise access to those quantities. A first one would be to properly scale the off-specular scattering intensities to the specular reflectivity, as described at the end of Sec. IV C, and to adjust the values of $\xi_{l}^{M}$ and $\xi_{l}^{R}$ in Eq. (22) and (25), respectively, in order to meet the measured intensity levels. A second method is to use another experimental geometry, namely the grazing incidence small angle neutron scattering (GISANS) geometry. In this geometry, the beam at sample position also arrives with a good collimation along the $y$ axis of Fig. 1, allowing one to resolve the angle $\varphi$ and therefore to measure the correlations along the $y$ axis. The component of the scattering wave vector $\mathbf{Q}$ along the $y$ axis can be made much larger than the one along the $x$ axis allowing one to probe lateral correlations on a smaller length scale. In that case, the correlation lengths are accessed by adjusting peak widths to the measured data. A first report of the GISANS investigation of the remagnetization process in the system considered here can be found in Ref. 78, and a full publication with simulation of the data is in preparation.

\section{ACKNOWLEDGMENTS}

We would like to thank P. Böni (Technische Universität München) for fruitful discussions on the subject of $\mathrm{FeCoV} / \mathrm{TiN}$ remanent polarizing supermirrors and SwissNeutronics for communicating the layer sequence of the sample.
${ }^{1}$ Review: P. Grünberg, J. Phys.: Condens. Matter 13, 7691 (2001).

${ }^{2}$ J. Nogues and I. K. Schuller, J. Magn. Magn. Mater. 192, 203 (1999).

${ }^{3}$ J. I. Martin, J. Nogues, K. Liu, J. L. Vicent, and I. K. Schuller, J. Magn. Magn. Mater. 256, 449 (2003).

${ }^{4}$ C. A. Ross, S. Haratani, F. J. Castano, Y. Hao, M. Hwang, M. Shima, J. Y. Cheng, B. Vogeli, M. Farhoud, M. Walsh, and H. I. Smith, J. Appl. Phys. 91, 6848 (2002).

${ }^{5}$ S. Sun, C. B. Murray, D. Weller, L. Folks, and A. Moser, Science 287, 1989 (2000).
${ }^{6}$ J. Y. Cheng, W. Jung, and C. A. Ross, Phys. Rev. B 70, 064417 (2004).

${ }^{7}$ R. Skomski, J. Phys.: Condens. Matter 15, R841 (2003).

${ }^{8}$ S. I. Woods, J. R. Kirtley, S. Sun, and R. H. Koch, Phys. Rev. Lett. 87, 137205 (2001).

${ }^{9}$ H. J. Richter, A. Y. Dobin, R. T. Lynch, D. Weller, R. M. Brockie, O. Heinonen, K. Z. Gao, J. Xue, R. J. M. v. d. Veerdonk, P. Asselin, and M. F. Erden, Appl. Phys. Lett. 88, 222512 (2006).

${ }^{10}$ T. Dietl, Nat. Mater. 5, 673 (2006).

${ }^{11}$ M. Jamet, A. Barski, T. Devillers, V. Poydenot, R. Dujardin, P. 
Bayle-Guillemaud, J. Rothman, E. Bellet-Amalric, A. Marty, J. Cibert, R. Mattana, and S. Tatarenko, Nat. Mater. 5, 653 (2006).

${ }^{12}$ A. Schreyer, C. F. Majkrzak, Th. Zeidler, T. Schmitte, P. Bödeker, K. Theis-Bröhl, A. Abromeit, J. A. Dura, and T. Watanabe, Phys. Rev. Lett. 79, 4914 (1997).

${ }^{13}$ J. A. Borchers, J. A. Dura, J. Unguris, D. Tulchinsky, M. H. Kelley, C. F. Majkrzak, S. Y. Hsu, R. Loloee, W. P. PrattJr, and J. Bass, Phys. Rev. Lett. 82, 2796 (1999).

${ }^{14}$ M. R. Fitzsimmons, P. Yashar, C. Leighton, I. K. Schuller, J. Nogues, C. F. Majkrzak, and J. A. Dura, Phys. Rev. Lett. 84, 3986 (2000).

${ }^{15}$ W.-T. Lee, S. G. E. te Velthuis, G. P. Felcher, F. Klose, T. Gredig, and E. D. Dahlberg, Phys. Rev. B 65, 224417 (2002).

${ }^{16}$ A. Paul, E. Kentzinger, U. Rücker, and Th. Brückel, Phys. Rev. B 74, 054424 (2006).

${ }^{17}$ K. V. O’Donovan, J. A. Borchers, C. F. Majkrzak, O. Hellwig, and E. E. Fullerton, Phys. Rev. Lett. 88, 067201 (2002).

${ }^{18}$ J. Stahn, J. Chakhalian, Ch. Niedermayer, J. Hoppler, T. Gutberlet, J. Voigt, F. Treubel, H.-U. Habermeier, G. Cristiani, B. Keimer, and C. Bernhard, Phys. Rev. B 71, 140509(R) (2005).

${ }^{19}$ A. Hoffmann, S. G. E. te Velthuis, Z. Sefrioui, J. Santamaria, M. R. Fitzsimmons, S. Park, and M. Varela, Phys. Rev. B 72, 140407(R) (2005).

${ }^{20}$ S. Bedanta, E. Kentzinger, O. Petracic, W. Kleemann, U. Rücker, Th. Brückel, A. Paul, S. Cardoso, and P. P. Freitas, Phys. Rev. B 74, 054426 (2006).

${ }^{21}$ S. Langridge, J. Schmalian, C. H. Marrows, D. T. Dekadjevi, and B. J. Hickey, Phys. Rev. Lett. 85, 4964 (2000).

${ }^{22}$ M. R. Fitzsimmons, S. D. Bader, J. A. Borchers, G. P. Felcher, J. K. Furdyna, A. Hoffmann, J. B. Kortright, I. K. Schuller, T. C. Schulthess, S. K. Sinha, M. F. Toney, D. Weller, and S. Wolf, J. Magn. Magn. Mater. 271, 103 (2004).

${ }^{23}$ V. Lauter-Pasyuk, H. J. Lauter, B. P. Toperverg, L. Romashev, and V. Ustinov, Phys. Rev. Lett. 89, 167203 (2002).

${ }^{24}$ K. Theis-Bröhl, M. Wolff, A. Westphalen, H. Zabel, J. McCord, V. Höink, J. Schmalhorst, G. Reiss, T. Weiss, D. Engel, A. Ehresmann, U. Rücker, and B. P. Toperverg, Phys. Rev. B 73, 174408 (2006).

${ }^{25}$ H. Zabel, Mater. Today 9, 42 (2006).

${ }^{26}$ C. Fermon, F. Ott, B. Gilles, A. Marty, A. Menelle, Y. Samson, G. Legoff, and G. Francinet, Physica B 267-268, 162 (1999).

${ }^{27}$ Review: J. F. Ankner and G. P. Felcher, J. Magn. Magn. Mater. 200, 741 (1999).

${ }^{28}$ Review: C. F. Majkrzak, Physica B, 221, 342 (1996).

${ }^{29}$ C. Fermon, Physica B 213\&214, 910 (1995).

${ }^{30}$ B. P. Toperverg, Physica B 297, 160 (2001).

${ }^{31}$ B. P. Toperverg, in Polarized Neutron Scattering, Series "Matter and Materials," Vol. 12, p. 247 (Forschungszentrum Jülich GmbH, Jülich, 2002).

${ }^{32}$ V. Lauter-Pasyuk, H. J. Lauter, B. Toperverg, O. Nikonov, E. Kravtsov, M. A. Milyaev, L. Romashev, and V. Ustinov, Physica B 283, 194 (2000).

${ }^{33}$ A. Paul, E. Kentzinger, U. Rücker, D. E. Bürgler, and T. Brückel, Phys. Rev. B 73, 094441 (2006).

${ }^{34}$ P. Böni, D. Clemens, M. Senthil Kumar, and S. Tixier, Physica B 241-243, 1060 (1998).

${ }^{35}$ G. Herzer, J. Magn. Magn. Mater. 112, 258 (1992).

${ }^{36}$ D. Clemens, A. Vananti, C. Terrier, P. Böni, M. Senthil Kumar, and M. Horisberger, J. Magn. Magn. Mater. 177-181, 1237 (1998).
${ }^{37}$ G. Pan and H. Du, J. Appl. Phys. 93, 5498 (2003).

${ }^{38}$ M. C. Contreras, J. F. Calleja, R. Matarranz, B. Presa, J. A. Corrales, and G. Pan, J. Appl. Phys. 99, 08F110 (2006).

${ }^{39}$ M. Senthil Kumar and P. Böni, J. Appl. Phys. 91, 3750 (2002).

${ }^{40}$ L. G. Parratt, Phys. Rev. 95, 359 (1954).

${ }^{41}$ L. Landau and E. Lifschitz, Mécanique Quantique, 3rd ed. (Mir, Moscou, 1975).

${ }^{42}$ O. Schärpf, in Polarized Neutron Scattering, Series "Matter and Materials," Vol. 12, p. 31 (Forschungszentrum Jülich GmbH, Jülich, 2002).

${ }^{43}$ M. Born and E. Wolf, Principles of Optics, 6th corr. ed. (Pergamon Press, New York, 1989).

${ }^{44}$ E. Kentzinger, U. Rücker, and B. Toperverg, Physica B 335, 82 (2003).

${ }^{45}$ L. Nevot and P. Croce, Rev. Phys. Appl. 15, 761 (1980).

${ }^{46}$ B. Vidal and P. Vinvent, Appl. Opt. 23, 1794 (1984).

${ }^{47}$ S. K. Sinha, E. B. Sirota, S. Garoff, and H. B. Stanley, Phys. Rev. B 38, 2297 (1988).

${ }^{48}$ A. Yoneda, Phys. Rev. 131, 2010 (1963).

${ }^{49}$ G. H. Vineyard, Phys. Rev. B 26, 4146 (1982).

${ }^{50}$ R. Pynn, Phys. Rev. B 45, 602 (1992).

${ }^{51}$ V. Holý, J. Kubena, I. Ohlídal, K. Lischka, and W. Plotz, Phys. Rev. B 47, 15896 (1993).

${ }^{52}$ V. Holý and T. Baumbach, Phys. Rev. B 49, 10668 (1994).

${ }^{53}$ J. Stettner, Ph.D. dissertation, Christian-Albrechts-Universität zu Kiel, 1995.

${ }^{54}$ O. Halpern and M. H. Johnson, Phys. Rev. 55, 898 (1939).

${ }^{55}$ R. M. Moon, T. Riste, and W. C. Koehler, Phys. Rev. 181, 920 (1969).

${ }^{56}$ J. F. Löffler, H. B. Braun, and W. Wagner, Phys. Rev. Lett. 85, 1990 (2000).

${ }^{57}$ A. Michels, R. N. Viswanath, J. G. Barker, R. Birringer, and J. Weissmüller, Phys. Rev. Lett. 91, 267204 (2003).

${ }^{58}$ J. Weissmüller, A. Michels, D. Michels, A. Wiedenmann, C. E. KrillIII, H. M. Sauer, and R. Birringer, Phys. Rev. B 69, 054402 (2004).

${ }^{59}$ E. Kentzinger, U. Rücker, B. Toperverg, and Th. Brückel, Physica B 335, 89 (2003).

${ }^{60}$ J. Stettner, L. Schwalowsky, O. H. Seeck, M. Tolan, W. Press, C. Schwarz, and H. v. Känel, Phys. Rev. B 53, 1398 (1996).

${ }^{61}$ D. R. Lee, S. K. Sinha, D. Haskel, Y. Choi, J. C. Lang, S. A. Stepanov, and G. Srajer, Phys. Rev. B 68, 224409 (2003).

${ }^{62}$ D. R. Lee, S. K. Sinha, C. S. Nelson, J. C. Lang, C. T. Venkataraman, G. Srajer, and R. M. Osgood III, Phys. Rev. B 68, 224410 (2003).

${ }^{63}$ J. P. Schlomka, M. Tolan, L. Schwalowsky, O. H. Seeck, J. Stettner, and W. Press, Phys. Rev. B 51, 2311 (1995).

${ }^{64}$ J. B. Hayter and H. A. Mook, J. Appl. Crystallogr. 22, 35 (1989).

${ }^{65}$ M. Senthil Kumar, P. Böni, and M. Horisberger, Physica B 325, 401 (2003).

${ }^{66}$ M. Senthil Kumar, V. R. Shah, C. Shanzer, P. Böni, T. Krist, and M. Horisberger, Physica B 350, e241 (2004).

${ }^{67}$ C. Schanzer, V. R. Shah, T. Gutberlet, M. Gupta, P. Böni, and H. B. Braun, Physica B 356, 46 (2005).

${ }^{68}$ C. Schanzer, Ph.D. dissertation, Technischen Universität München, 2006.

${ }^{69}$ U. Rücker, E. Kentzinger, B. Toperverg, F. Ott, and T. Brückel, Appl. Phys. A: Mater. Sci. Process. 74, S607 (2002).

${ }^{70}$ U. Rücker, B. Alefeld, W. Bergs, E. Kentzinger, and T. Brückel, Physica B 276-278, 95 (2000). 
${ }^{71}$ U. Rücker, W. Bergs, B. Alefeld, E. Kentzinger, and T. Brückel, Physica B 297, 140 (2001).

${ }^{72}$ http://www.fz-juelich.de/iff/wns_hadas

${ }^{73}$ U. Rücker (unpublished).

${ }^{74}$ N. K. Pleshanov, N. G. Kolyvanova, S. V. Metelev, B. G. Peskov, V. M. Pusenkov, V. G. Syromyatnikov, V. A. Ul'yanov, and A. F. Schebetov, Physica B 369, 234 (2005).
${ }^{75}$ G. Herzer, J. Magn. Magn. Mater. 294, 99 (2005).

${ }^{76} \mathrm{G}$. Bertotti, Hysteresis in Magnetism (Academic, New York, 1998).

${ }^{77}$ R. Alben, J. J. Becker, and M. C. Chi, J. Appl. Phys. 43, 1653 (1978).

${ }^{78}$ E. Kentzinger, H. Frielinghaus, U. Rücker, A. Ioffe, D. Richter, and Th. Brückel, Physica B 397, 43 (2007). 